\title{
Reactant Jetting in Unstable Detonation
}

\author{
S.R. Sanderson ${ }^{1}$, J.M. Austin ${ }^{2}$, Z. Liang ${ }^{3}$, F. Pintgen ${ }^{4}$, J.E. Shepherd, \\ H.G. Hornung \\ Alumni, Faculty and Director Emeritus of \\ Graduate Aeronautical Laboratories, California Institute of Technology, \\ Pasadena, CA, 91125, USA.
}

In Honor of Professor Hornung's 75th Birthday.

[ Appears in Prog Aerospace Sci (2009), doi:10.1016/j.paerosci.2009.11.002 ]

\begin{abstract}
We note the common existence of a supersonic jet structure locally embedded within a surrounding transonic flow field in the hitherto unrelated phenomena of unstable gaseous detonation and hypervelocity blunt body shock wave interaction. Extending prior results that demonstrate the consequences of reduced endothermic reaction rate for the supersonic jet fluid in the blunt body case, we provide an explanation for observations of locally reduced $\mathrm{OH}$ PLIF signal in images of the keystone reaction zone structure of weakly unstable detonations. Modeling these flow features as exothermically reacting jets with similarly reduced reaction rates, we demonstrate a mechanism for jetting of bulk pockets of unreacted fluid with potentially differing kinetic pathways into the region behind the primary detonation front of strongly unstable mixtures. We examine the impact of mono-atomic and diatomic diluents on transverse structure. The results yield insight into the mechanisms of transition and characteristic features of both weakly and strongly unstable mixtures.
\end{abstract}

\footnotetext{
${ }^{1}$ Current address: General Electric, 12345 SW Leveton Drive, Tualatin OR 97062.

${ }^{2}$ Current address: University of Illinois at Urbana-Champaign, Urbana IL 61801.

${ }^{3}$ Current address: AECL Chalk River Laboratories, Chalk River, Ontario K0J 1J0.

${ }^{4}$ Current address: General Electric, 3000 N Grandview Blvd, Waukesha WI 53188.
} 


\section{Introduction}

\subsection{Structure of Paper}

Our goal in the current paper is to demonstrate the consequences of the local structural equivalence of two analogous flow fields. To provide the necessary context, we begin with a brief summary of recent developments in the state of knowledge for the two flow fields, each treated separately and without reference to the consequences of the material that follows. We then demonstrate the local equivalence of the two flow fields and examine the potential implications. Subsequent sections develop a model that draws on existing knowledge from both fields that we use to aid interpretation of phenomena observed in experimental detonation data.

\subsection{Reaction Zone Structure in Unstable Detonation}

Austin [1,2] describes experiments that investigate the consequences of the strong temperature dependence of chemical kinetics on the reaction zone structure of unstable gaseous detonations. Experiments were conducted over a range of fuel-oxidizer-diluent mixtures that were expected to induce varying degrees of instability, according to existing models. Superposed images of shock fronts and reaction zone structures reveal new details of the coupling between flow instability and reaction processes. Note also the review article of Shepherd [3] and Radulescu et al [4].

Gas mixtures are generally categorized as either weakly or strongly unstable, depending upon the observed behavior of the detonation. In the case of weakly unstable mixtures, the flow field is characterized by a regular cellular structure (Figure 1(a),(b); Schlieren images). Adjacent cells of the structure exhibit advancing and retreating main shock fronts respectively, relative to the mean detonation front velocity. Pairs of counter propagating transverse waves, $\mathrm{T}^{+}$and $\mathrm{T}^{-}$, intersect each segment of the advancing main front, A, where it joins with the segments of the retreating front above and below it, R. The boundaries of the detonation cells are traced out by the path of complex shock wave interaction structures formed at the wave junctions (Figures 2(a),(b)). Measurements of the reaction zone structure using planar laser-induced fluorescence (PLIF) imaging of the $\mathrm{OH}$ radical reveal a reaction zone structure with a characteristic "key-stone" shape [5] (Figure 1(a),(b); OH PLIF images) that remains tightly coupled to the advancing main shock fronts but lags the retreating main shock fronts. Despite the widened induction zone behind the retreating main shock front, the flow 
remains essentially laminar and the reaction zone may be described by simple models that describe the species concentrations along streamlines. Austin uses chemical kinetics models to explain the observed key-stone images but does however note some inconsistency between the observed transverse wave angle and that predicted by shock triple point calculations at the intersection of the transverse wave with the main detonation front.

In contrast to the regular cellular structure of weakly unstable mixtures, observations of strongly unstable mixtures reveal detonations with a highly convoluted reaction zone structure (Figure 1(c),(d); OH PLIF images) behind an irregular shock front (Figure 1(c),(d); Schlieren images) and produce a soot foil with a broader range of length scales (Figure 2(c),(d)).

Detailed calculations of induction times behind the various waves at the standard triple point structure apparently formed by the transverse waves reveal no significant trends in mixture reactivity that would explain the data for the various weakly and strongly unstable mixtures. Austin goes on to study the applicability of the critical decay rate model developed by Eckett et al [6] that provides predictive estimates of the main shock front unsteadiness required to decouple the reaction zone from the gas dynamic shock wave and its relationship to observed transitions in weakly and strongly unstable behavior. It is surprising however that although the critical decay rate model predicts transition mechanisms rooted in the decoupling of a decelerating wave as it proceeds along each subsequent cell, large scale turbulent structures are also observed in OH PLIF images of the reaction zone structure behind the stronger advancing portion of the front. Further study of the range of scales produced in highly unstable detonation leads Austin to speculate on a characterization of detonation stability regimes based on a two dimensional parameter space based on relative velocity fluctuation $u^{\prime} / U_{C J}$ and relative induction time fluctuation $\tau^{\prime} / \tau_{C J}$, analogous to the Borghi diagram in turbulent combustion.

Subsequent efforts [7] have examined the appearance of hot spots, localized explosions, and the influence of hot product/cold reactant diffusion across the shear layer produced by the intersection of a transverse wave with the main shock front. In mixtures with low effective activation energy, vortical structures associated with Kelvin-Helmholtz instability were observed to occur, but only downstream of the ignition. In mixtures with high activation energy, a high speed transverse reaction front was found to develop near the shear layer interface spreading ignition into the cold stream. 

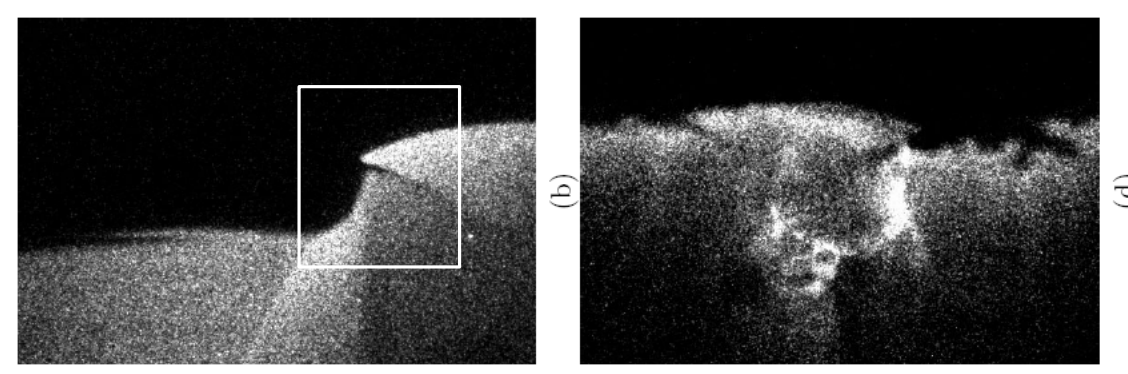

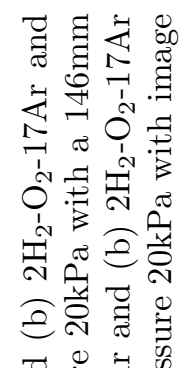
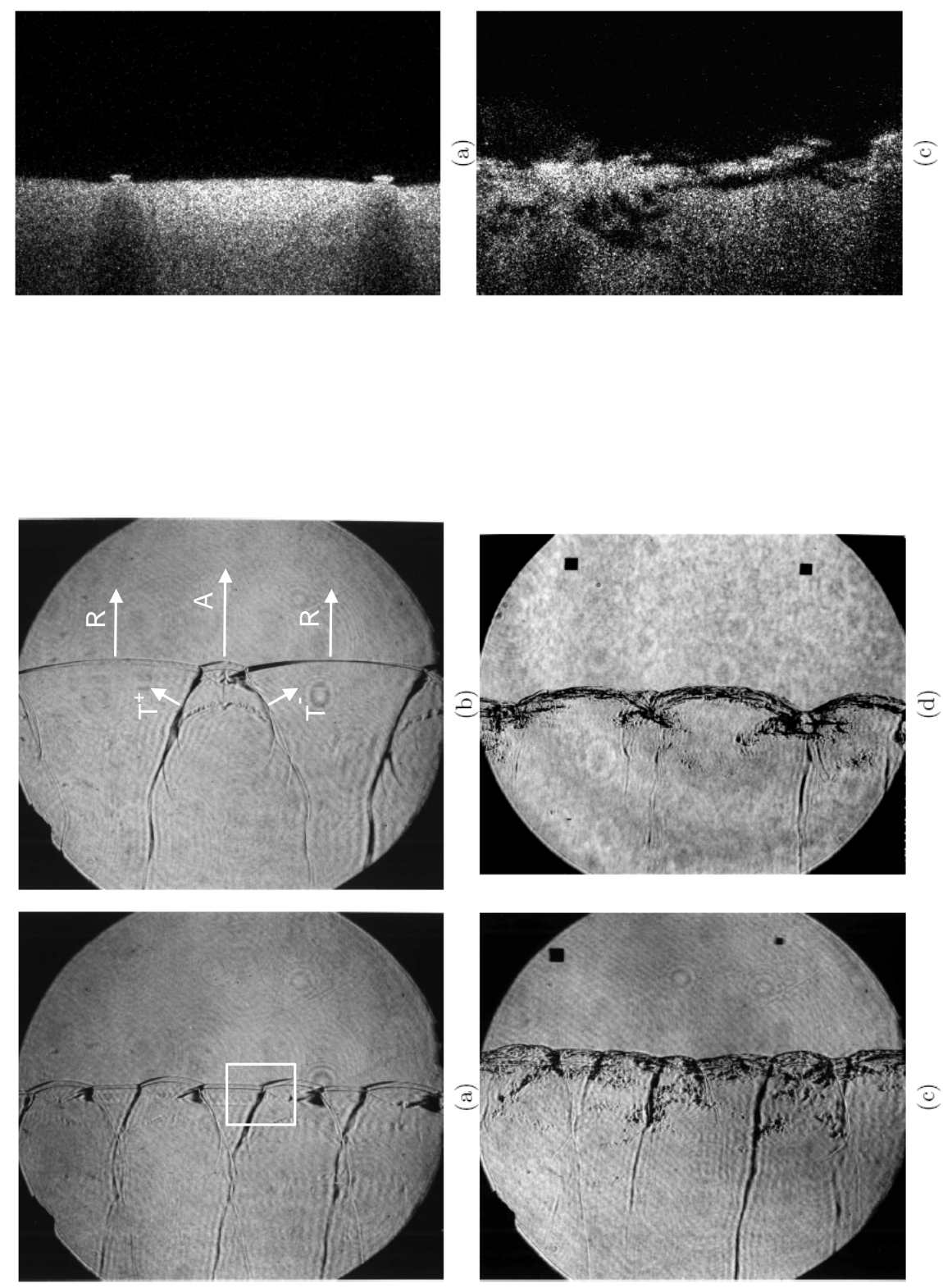

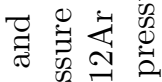

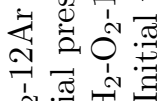

ஸे. 空 ฮై

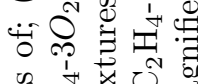

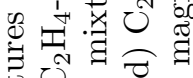
. घ 요 चี ヨ च ฮี त. 芯苋方

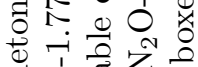
षैं क्ष

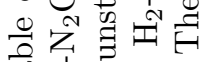

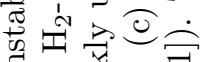
可范藏

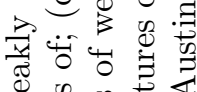

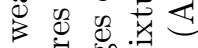

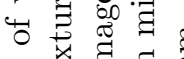
के . . . ह 四

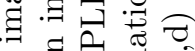

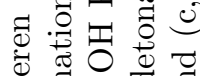

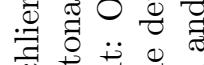

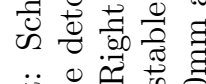

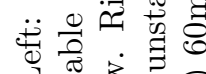

个造兘

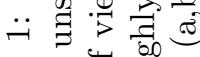

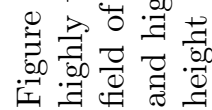




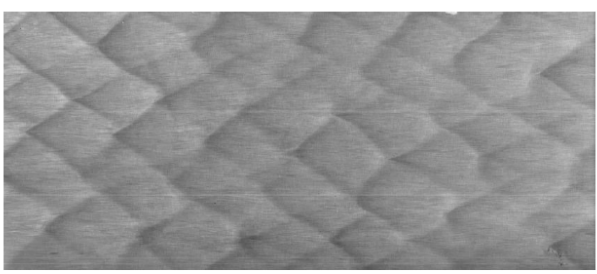

(a)

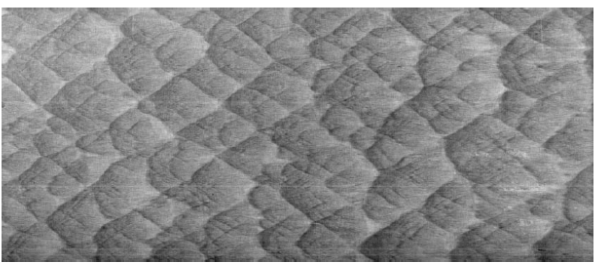

(c)

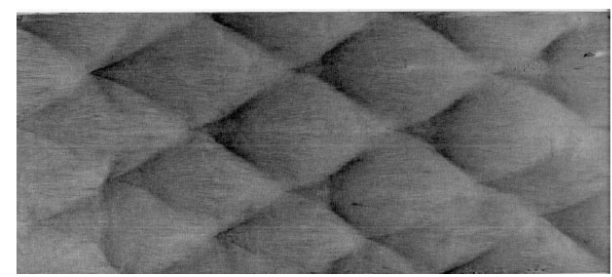

(b)

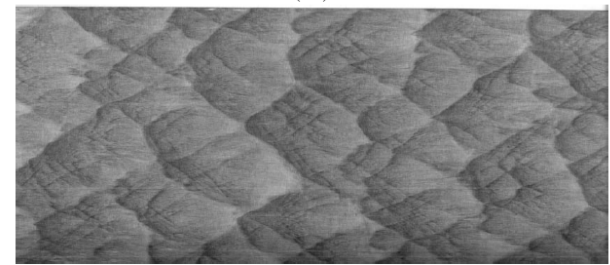

(d)

Figure 2: Soot foil visualization of the paths of shock wave intersections for weakly unstable detonation in mixtures of; (a) $2 \mathrm{H}_{2}-\mathrm{O}_{2}-12 \mathrm{Ar}$ and (b) $2 \mathrm{H}_{2}-\mathrm{O}_{2}-17 \mathrm{Ar}$ and highly unstable detonation in mixtures of; (c) $\mathrm{H}_{2}-\mathrm{N}_{2} \mathrm{O}-1.33 \mathrm{~N}_{2}$ and (d) $\mathrm{C}_{3} \mathrm{H}_{8}-5 \mathrm{O}_{2}-9 \mathrm{~N}_{2}$. Initial pressure $20 \mathrm{kPa}$ with imaged soot foil height $=152 \mathrm{~mm}$. (Austin [1]).

\subsection{Shock Impingement on a Blunt Body in Steady Hypervelocity Flow}

Sanderson $[8,9]$ describes the physical model (Figure 3) of the Type IV shock interaction flow field that was originally proposed by Edney [10, 11]. In figure 3 a three shock $\lambda$-pattern is observed at the point where the impinging shock wave, 1 , interacts with the bow shock, 2. A strong vortex sheet emanates from the $\lambda$-point. The shock layer flow above the vortex sheet is subsonic whereas the flow between the vortex sheet and reflected shock wave, 3 , remains supersonic. A second, less distinct, inverted $\lambda$-pattern is observed at the intersection between the reflected shock, 3 , and the continuation of the strong bow shock below the interaction region, 4 . An additional oblique wave, 5 , is reflected upwards at the secondary $\lambda$-point and a second vortex sheet is produced. Observe that the two vortex sheets bound a supersonic jet that is embedded in the surrounding subsonic shock layer. The jet is formed from the fluid passing between the two $\lambda$-points. The oblique wave, 5 , crossing the jet is guided along the supersonic jet by successive reflections from the two shear layers. The inviscid jet structure is gradually consumed by the spreading of the two shear layers that entrain the surrounding subsonic shock layer fluid. 

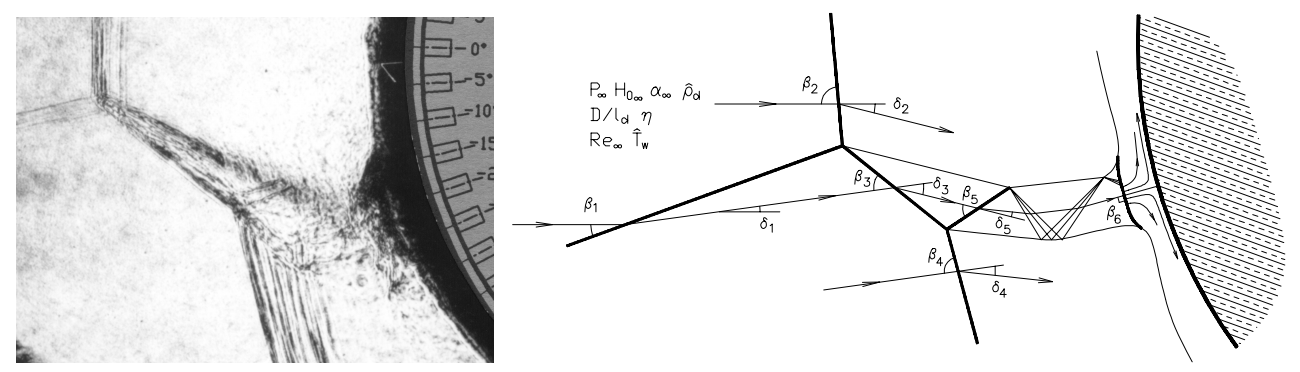

Figure 3: Holographic shadowgraph image (left) and physical model (right) of the type IV impingement of an oblique shock wave near the geometrical stagnation point of a cylinder in hypersonic flow. The free stream parameters shown in the physical model for the case of an ideal dissociating gas are defined in section 3. For a perfect gas this set of parameters reduces to $M_{\infty}$ and $\gamma$. Solid lines represent shock waves 1-5 and are identified by the indicated shock wave angles, $\beta_{1-5}$ and flow deflection angles, $\delta_{1-5}$. The half weight lines that originate at the two mutually inverted $\lambda$-shock wave intersections represent the shear layers that originate at these two locations. Note also the free streamline reflection of shock 5 along the length of the jet, along with the stagnation streamline that passes through the center of the jet in the type IV configuration. (Sanderson [8]: $M_{\infty}=9.9, \mathrm{~N}_{2}$ at $h_{0}=3.9$ $\mathrm{MJ} / \mathrm{kg})$.

Again studying the triple point structure formed in the vicinity of the shock interaction, local flow field analysis demonstrates that given only the free stream conditions and the incident shock angle, $\beta_{1}$, all of the wave angles and flow properties at both $\lambda$-points and along the length of the supersonic jet are known. It is remarkable that all local features of the flow are thereby completely determined, up to an unknown length scale. Sanderson et al $[13,12]$ demonstrate that beyond smooth regions of the main shock front, a strictly limited set of possible solutions are admissible at discontinuities on the shock front, even for vanishingly weak disturbances. The global flow field therefore adapts to accommodate the existence of the jet flow structure at some length scale determined by interaction of the local and global scales.

Sanderson et al [9] demonstrate via experiment and further modeling that peak heating, caused by the impingement of the jet on the body, occurs when the strengths of the oblique shock waves (1,3 and 5) in the supersonic jet are balanced, with no single wave of excessive strength. This minimizes the entropy rise along the stagnation streamline that passes between the two $\lambda$-points and downstream through the supersonic jet. This minimum entropy condition concomitantly depresses the dissociative reaction rate of the jet fluid in the case of hypervelocity flow, since temperature decreases with 
decreased entropy at constant pressure as determined by the surrounding subsonic shock layer. At Mach numbers in the range, $M_{\infty}=5-10$, Sanderson et al [9] demonstrate a $10-100 \times$ decrease in the dissociation rate of the jet fluid (driven by reduced temperature and density) relative to the undisturbed shock layer fluid, underscoring the magnitude of the effect.

These conclusions of suppressed jet reaction rate are supported by the numerical simulations of both Brück [14] and Carlson and Wilmoth [15] using continuum and DSMC based models respectively.

\section{Equivalence of Unstable Detonation and Steady Flow Shock In- teraction Shock Wave Systems}

\subsection{Structural Equivalence}

In the current paper we re-examine the role of the detailed shock interaction structures formed at the cell boundaries and the potential influence on detonation stability. Adopting the reference frame approach used to relate the treatment of steady flow and self-similar Mach reflection problems [16], we begin by considering the relationship between the previously unrelated phenomena of sections 1.2 and 1.3. By transforming the detonation data into a moving reference frame with horizontal and vertical velocity components matching the main detonation front speed, $U_{M}$, and transverse wave speed, $U_{T}$, respectively, and by subsequently rotating the moving reference frame through an angle that matches the shock intersection track angle, $\tan \phi=U_{T} / U_{M}$, the local equivalence of the two flow fields becomes apparent (see figure 4). The most visually striking similarity is the duplication

of the double, mutually inverted, $\lambda$-point structures in the CFD results of Liang et al [17] for weakly unstable detonation in comparison with the type IV interaction data. Noting the suppressed reaction rate for the jet fluid in the type IV interaction discussed previously, it is reasonable to propose that the previously unexplained "fracture" (that appears as a nearly horizontal dark feature in this reference frame) in the corner of the OH PLIF keystone reaction zone suggests the presence of a cold jet in the detonation case also. The ability of 2D CFD to visualize disparate length scales in the flow-field is of great utility here, complementing the experimental Schlieren data that has inevitably primacy and reveals a self consistent far-field structure, but suffers from the unavoidable difficulty of imaging fine flow field features in the presence of three dimensional structure and very strong aero-optical density gradients. No discrete comparison between the images comprising figure 4 
is conclusive, yet the separate clues combine as a group to provide strong evidence of a local structural equivalence between unstable detonation and type IV shock wave interaction.

\subsection{Implications for Transverse Wave Structure}

The detailed structure of the transverse wave interaction zone has been investigated by a number of authors (see for example [18, 19, 20, 21, 22]). Simple three wave interaction calculations do not always produce consistent results that reconcile experimentally observed wave angles. Although prior efforts to develop strong triple point configurations that incorporate multiple wave interactions address some of the difficulties, the current ensemble data in combination with the realization of the relevance of the physical model originally advanced by Edney [10] inform a modified physical model of the unstable detonation transverse wave structure (Figure 5). Rather than adjust the form of the model, study of the shock wave interaction problem suggests rather that the scale of the interaction is variable relative to the surrounding flow, with the basic form being preserved since it is locally required by the conservation law requirements at the shock bifurcation points, irrespective of the distortion of the surrounding flow.

\subsection{Implications for Jet Reaction Kinetics}

Beyond the similarity in wave structure between detonation and shock wave interaction, there are further parallels in the effects of non-equilibrium flow thermochemistry. The key-stone pattern OH PLIF images of Pintgen et al [5] and Austin [1, 2], the thin band of unreacted fluid noted in the species concentration profiles predicted by Liang et al [17], and Sanderson's $[9,8]$ conclusions of a greatly suppressed jet reaction rate, all combine with the relationship of the local shock structures, to suggest that the jetting mechanism observed in the hypervelocity blunt body case, when reduced to a sufficiently small scale, provides an explanation for the apparent "fracture" in the keystone PLIF images that is generally observed in the weakly unstable cases of Austin and Pintgen.

Extending the argument to the case of strongly unstable detonation, we propose that this represents a mechanism for the formation of a series of reactant jets along the main detonation front, each jet associated with a particular transverse wave, that propel quantities of unreacted fluid at relatively low temperature through the otherwise reaction inducing strong detonation front. The effect of the proposed jets would be to provide a mechanism for 


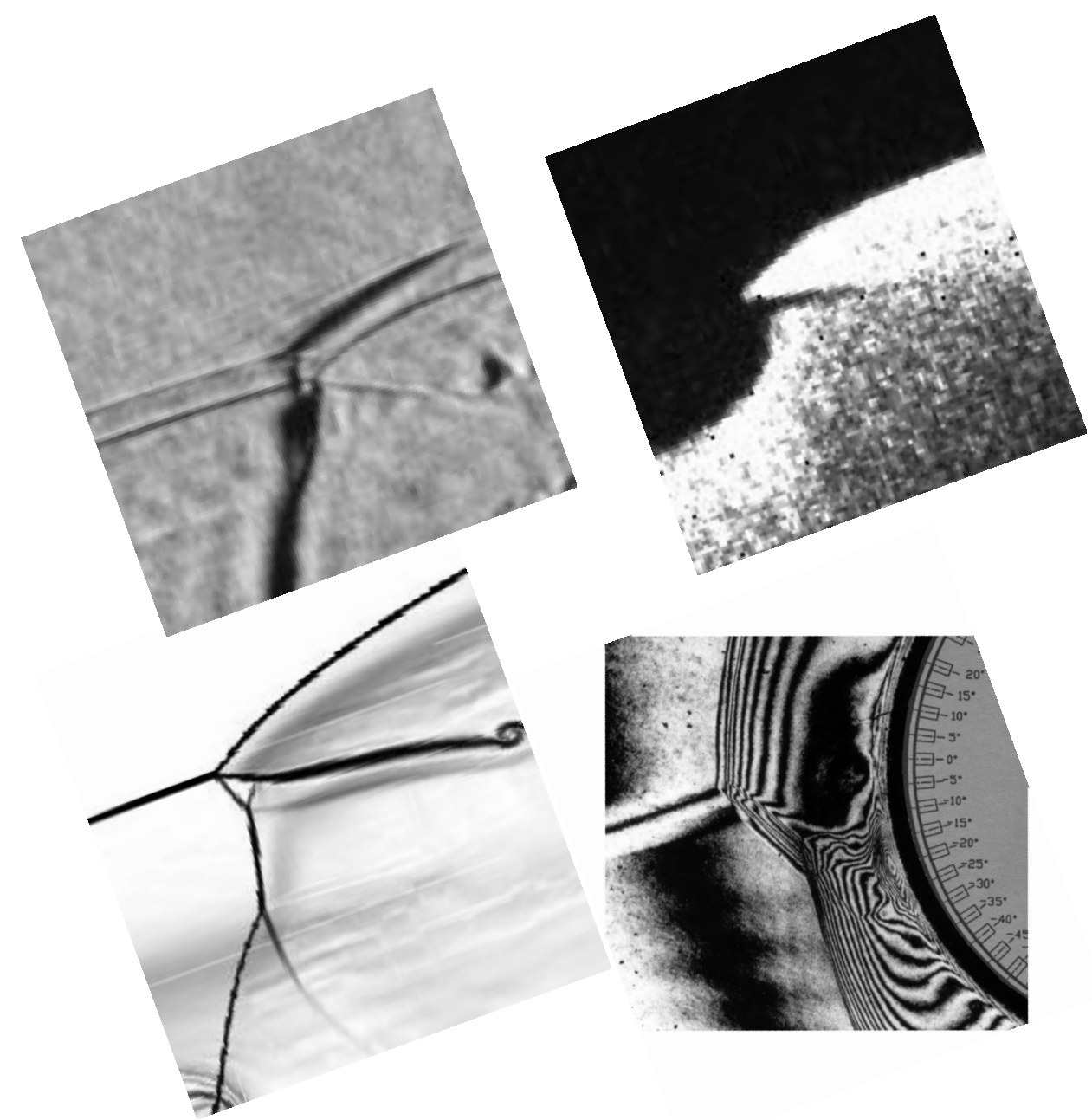

Figure 4: Equivalence of wave structures in weakly unstable detonation and steady flow shock interaction when transformed to a reference frame fixed with respect to the transverse wave and aligned with the incident flow direction. From left-right, top-bottom: Schlieren image of weakly unstable detonation in $2 \mathrm{H}_{2}-\mathrm{O}_{2}-12 \mathrm{Ar}$ at $20 \mathrm{kPa}$ initial pressure [1] (note the apparent "double" shock front structure in the Schlieren image results from inflection of the lead wave due to an out-of-plane transverse wave), OH PLIF image of weakly instable detonation in $2 \mathrm{H}_{2}-\mathrm{O}_{2}-17 \mathrm{Ar}$ at $20 \mathrm{kPa}$ initial pressure [1], Computational Schlieren image of weakly unstable pseudo-mixture [17], Holographic interferogram of hypervelocity chemical non-equilibrium Type IV interaction in $\mathrm{N}_{2}$ at $h_{0}=19 . \mathrm{MJ} / \mathrm{kg}$ [9]. The first three images are magnified and reoriented views of the boxed regions indicated in figures 1(a); Schlieren images, 1(b);OH PLIF images and 10; weak case respectively. The fourth image is an alternate interferometric visualization at high enthalpy conditions of the same flow field that is illustrated in figure 3. Note especially the low entropy jet fluid that results in the appearance of a region of greatly increased density at the jet impingement point on the body. 


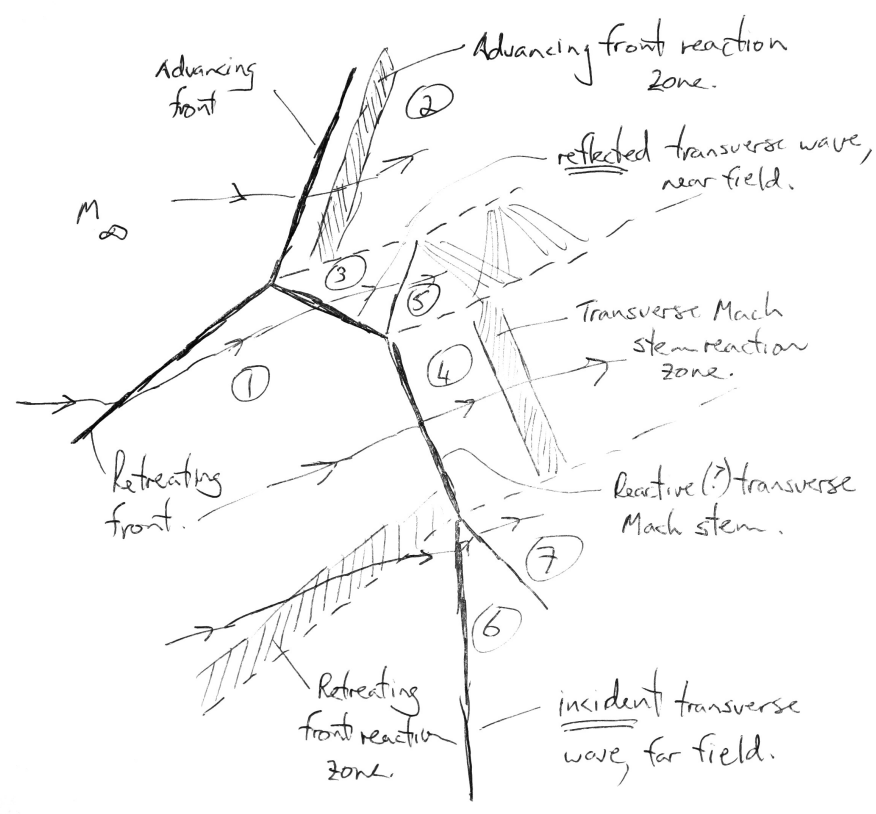

Figure 5: Proposed physical and chemical model of transverse wave structure in unstable detonation. (In the finest traditions of Prof. Hornung's many contributions; this work has its origins in improvised sketches and order of magnitude calculations shared with enthusiasm amongst collaborators. We leave this sketch in its original form, finding no real modification that would improve its meaning for the current purpose.)

the formation of the pockets of unreacted gas observed within the reaction zone in the strongly unstable detonation data.

Sanderson et al [23] show that due to the highly non-linear nature of the kinetics and oblique shock jump relationships, that both equilibrium and non-equilibrium thermo-chemical effects are largely confined to only the strongest waves (often nearly normal) in any given local flow field. This leads to approximation schemes for complex shock wave interactions where only the strongest waves in the shock structure are considered to be reactive (with infinitely fast chemistry), with the remainder considered to be frozen (with infinitely slow chemistry). The validity of the approximation is considered by computing the actual kinetic rates, based on the flow parameters for the approximate solution, with subsequent adjustment of the kinetic assumptions 
for the respective waves. Despite the possibility of a range of finite kinetic rates for which no valid approximation is possible, in practice the approximation is frequently meaningful and leads to useful conclusions. The proposed physical model (Figure 5) therefore reflects both the wave structures required for consistency with conservation equation requirements at the various shock wave intersections, along with appropriate assumptions regarding the frozen or reacted state of the various waves throughout the structure. Thus we seek kinetic scalings, in addition to the fluid mechanical scalings between the near and far field, that accommodate the necessity of the local existence of the jet structure.

Although the model can be proposed based on inspection of the experimental data (Figure 4), we follow with some analysis that helps explain the necessity and implications of various features of the physical model.

\section{Local Analysis about Discontinuities on the Detonation Front}

Local flow field analysis in the vicinity of the shock wave intersection points by mapping into the pressure-flow deflection angle $(p-\delta)$ plane is a standard methodology that has yielded valuable insight for various shock interaction phenomena. The fundamentals of the method are well known and have their basis in the study of Mach reflection (see Courant \& Friedrichs [24] and Hornung [16]). Analogous methods have found use in earlier studies of transverse waves in detonation $[18,19,20,21,22]$. We seek to relate these prior analyses to the apparently related phenomena investigated by Edney [10] and extended to the case of endothermically reacting flows by Sanderson et al [23], using analogous methods. Importantly, Edney's contributions were facilitated by effective instrumentation and imaging that enabled the synthesis of an adequate physical model of the shock interaction flow field for further analysis (Figure 3). Similarly, we proceed to analyze the proposed physical model of detonation transverse wave structure suggested by the data discussed above.

We begin by adapting the methodology of Sanderson et al [23] who systematized the methods originally elucidated by Hornung et al [25] for various configurations of endothermically reacting shock waves, to develop a compact dimensionless framework for the consistent treatment of normal, oblique and interacting detonation waves. We model the system as a dissociating diluent according to the thermo-chemical models of Lighthill [26] and Freeman [27] 
concerning the relaxation to equilibrium of the system,

$$
\begin{gathered}
\mathrm{D}_{2}+\mathrm{M} \rightleftharpoons \mathrm{D}+\mathrm{D}+\mathrm{M} \\
p=\frac{k}{2 m}(1+\alpha) \rho T \\
h=\frac{k}{2 m}\left[(4+\alpha) T+\alpha \theta_{d}\right]
\end{gathered}
$$

where $\mathrm{D}$ represents the diluent gas and $\mathrm{M}$ is a non-reacting third body. Here, $\rho$ is the density of the gas, $p$ is the pressure, $h$ is the enthalpy per unit mass, $k$ is Boltzmann's constant, $\mathrm{m}$ is the mass of one atom of the diluent, $T$ is the temperature, $\theta_{d}$ is a temperature characterizing the dissociation energy and $\alpha$ is the dissociated mass fraction determined from the number densities, $\alpha=\frac{n_{D}}{n_{D}+2 n_{D_{2}}}$.

We model ideal heat release according to an isenthalpic induction step with finite delay, $\tau$, followed by an infinitely fast heat release step, $\chi: 0 \rightarrow 1$, with addition of energy, characterized by temperature $\theta_{c}$, but no impact to species concentration,

$$
\chi=\left\{\begin{array}{l}
0 ; t<\tau \\
1 ; t \geq \tau
\end{array}\right.
$$

with $\tau=\frac{A}{\rho^{\mu}} \exp \frac{\theta_{a}}{T}$. For asymptotic consideration of the gas dynamic and kinetic scales, this degree of complexity is sufficient to establish the fundamental behavior. In order to understand the basic implications of the model, we initially take the pre-exponential density dependence, $\mu=1$, on the basis that the induction steps of relevant high temperature hydrocarbon and hydrogen reaction mechanisms are generally bimolecular in nature (note however the discussion of section 6.1).

\subsection{Normal Waves}

Consider initially a one dimensional normal wave. In dimensionless form, conservation of mass, momentum and energy across the wave gives,

$$
\begin{gathered}
\hat{\rho} \hat{u}=1 \\
\hat{p}=1+\frac{1}{P_{1}} \frac{\hat{\rho}-1}{\hat{\rho}} . \\
\left(4+\alpha_{2}\right) \frac{\hat{T}}{\Theta_{1}}+\alpha_{2}+\frac{K_{1}}{\hat{\rho}^{2}}=H_{0_{1}}+\left(\chi_{2}-\chi_{1}\right) \frac{\theta_{c}}{\theta_{d}}
\end{gathered}
$$


Additionally we define $u$ as the velocity normal to the wave, $h_{0}$ is the total enthalpy per unit mass. The fundamental changes to this formulation are the carrying through of the terms representing progress of the ideal heat release reaction, $\chi \in[0,1]$, that is characterized by temperature, $\theta_{c}$. The subscripts, 1 and 2, refer (contrary to prior use in the context of figures 3 and 5) to the upstream and downstream states respectively and generally the notation $\hat{\rho}=\rho_{2} / \rho_{1}$ applies.

The following parameterization has been used;

$$
\begin{gathered}
P_{1}=\frac{p_{1}}{\rho_{1} u_{1}^{2}}, \\
\Theta_{1}=\frac{\theta_{d}}{T_{1}}, \\
K_{1}=\frac{m u_{1}^{2}}{k \theta_{d}}, \\
H_{0_{1}}=\frac{2 m h_{0_{1}}}{k \theta_{d}},
\end{gathered}
$$

Note that $P_{1}$ behaves as $P_{1} \sim 1 / M_{1}^{2}$ for a perfect gas (see below). The conserved stagnation enthalpy, $H_{0_{1}}$, is normalized with respect to the dissociation energy of the gas and $K_{1}$ is the normalized specific kinetic energy of the upstream flow. For the case of a detonation wave, as currently modeled, four parameters are sufficient to define the state of the upstream gas and here we specify $P_{1}, H_{0_{1}}, \alpha_{1}$ and $\chi_{1}$. Sanderson et al [23] give the remaining parameters, $K_{1}, \Theta_{1}$, that are convenient forms used to simplify the notation, but dependent on the prior set.

$$
\begin{gathered}
\Theta_{1}=\frac{4+\alpha_{1}}{H_{0_{1}}-K_{1}-\alpha_{1}}, \\
K_{1}=\frac{H_{0_{1}}-\alpha_{1}}{1+2 P_{1} \frac{4+\alpha_{1}}{1+\alpha_{1}}} .
\end{gathered}
$$

After manipulation we obtain the following result that applies for an arbitrary non-equilibrium upstream state $\left(\alpha_{1} \neq 0, \chi_{1} \neq 0,1\right)$ and throughout the downstream non-equilibrium reaction zone,

$$
\left(H_{0_{1}}+\left(\chi_{2}-\chi_{1}\right) \frac{\theta_{c}}{\theta_{d}}-\alpha_{2}\right) \hat{\rho}^{2}-2 K_{1}\left(1+P_{1}\right) \frac{4+\alpha_{2}}{1+\alpha_{2}} \hat{\rho}+K_{1} \frac{7+\alpha_{2}}{1+\alpha_{2}}=0 .
$$


For a given upstream state, this result describes the evolution of the gas density across the downstream reaction zone, parameterized in terms of the dissociative and heat release reaction progress variables, $\alpha_{2}$ and $\chi_{2}$, whose rates of reaction remain free and unspecified. Note that two solution branches are given by this quadratic expression for the density, each familiar as the strong and weak detonation solutions respectively.

Expressing the induction delay (equation (4)) as a length scale, $\Delta=u \tau$ and non-dimensionalizing by a characteristic length based on the upstream flow, $\ell_{i_{1}}=u_{1} A / \rho_{1}^{\mu}$,

$$
\hat{\Delta}_{2}=\frac{\Delta_{2}}{\ell_{i_{1}}}=\hat{\rho}^{-(\mu+1)} e^{\frac{\Theta_{1}}{\hat{T}} \frac{\theta_{a}}{\theta_{d}}}
$$

The dissociation reaction similarly proceeds from the frozen von Neumann state downstream of the gas dynamic shock to the equilibrium state at rate,

$$
\frac{d \alpha_{2}}{d \hat{x}}=\ell_{d_{1}} \frac{d \alpha_{2}}{d x}=\hat{\rho}^{2} \hat{T}^{\eta} \Theta_{1}^{-\eta}\left[\left(1-\alpha_{2}\right) e^{\frac{-\Theta_{1}}{\hat{T}}}-\frac{\hat{\rho}}{\hat{\rho}_{d_{1}}} \alpha_{2}^{2}\right],
$$

with characteristic dissociation scale, $\ell_{d_{1}}=\frac{u_{1}}{C \rho_{1} \theta_{d}^{\eta}}$. In both cases (equations 15 and 16) the exponential temperature dependence is related to the evolution of the density field in $\rho(\alpha, \chi)$-space (equation (14)) according to,

$$
\frac{\Theta_{1}}{\hat{T}}=\frac{\hat{\rho}^{2}\left(1+\alpha_{2}\right)}{2 K_{1}\left(\hat{\rho}\left(P_{1}+1\right)-1\right)} .
$$

Here $A$ and $C$ are rate constants for the induction and dissociation reactions respectively, $\mu$ represents the density dependence and order of the induction step and $\eta$ represents the pre-exponential temperature dependence of the dissociation rate. In the equilibrium limit far downstream of the reaction zone the dissociation reaction asymptotes to,

$$
\frac{\alpha_{2_{e q}}^{2}}{1-\alpha_{2_{e q}}}=\frac{\hat{\rho}_{d_{1}}}{\hat{\rho}_{e q}} e^{\left.\frac{\left(1+\alpha_{2 q}\right) \hat{\rho}_{e q}^{2}}{2 K_{1}(1-\hat{\rho} e q}\left(1+P_{1}\right)\right)} .
$$

with the introduction of a dimensionless equilibrium constant,

$$
\hat{\rho}_{d_{1}}=\rho_{d} / \rho_{1} .
$$

In order to study the implications of the CJ condition in complex detonation wave interactions note that the speed of sound in the case of frozen chemistry is given by,

$$
a_{f}^{2}=\frac{k}{2 m} \frac{(1+\alpha)(4+\alpha)}{3} T
$$


so that in the dimensionless notation used here,

$$
M_{f}^{2}=\frac{u^{2}}{a_{f}^{2}}=\frac{3}{(4+\alpha) P} .
$$

The above results apply throughout the non-equilibrium reaction zone for straight shock waves. Typical values of the physical constants for the IDG model of nitrogen are given in table 1.

\subsection{Parameters Downstream of Oblique Waves}

The extension to oblique waves follows Sanderson et al [23]. If $\beta$ is the angle of the oblique shock wave, then the wave normal components of the upstream parameters become,

$$
\begin{gathered}
P_{1_{N}}=\frac{p_{1}}{\rho_{1} u_{1}^{2} \sin ^{2} \beta}=\frac{P_{1}}{\sin ^{2} \beta}, \\
K_{1_{N}}=\frac{m u_{1}^{2} \sin ^{2} \beta}{k \theta_{d}}=K_{1} \sin ^{2} \beta .
\end{gathered}
$$

The subscripts $N$ and $T$ refer to normal and tangential components respectively. The identity (13) then gives the dimensionless total enthalpy based on the shock normal components,

$$
H_{0_{1_{N}}}=K_{1_{N}}\left(1+2 P_{1_{N}} \frac{4+\alpha_{1}}{1+\alpha_{1}}\right)+\alpha_{1} .
$$

The reaction zone solution then follows according to equation (14) based on the normal components given above. Given the density profile across the reaction zone, the mapping of the streamline into the $p$ - $\delta$ plane is given by equation (6) for the pressure and the flow deflection is determined from continuity and invariance of tangential velocity across the shock to be,

$$
\tan (\beta-\delta)=\frac{\tan \beta}{\hat{\rho}}
$$

To facilitate the computation of complex wave interaction problems, convenient forms are required for the downstream dimensionless parameters $P_{2}$, $H_{0_{2}}$ and $\frac{\hat{\rho}_{d_{2}}}{\hat{\rho}_{d_{1}}}$. Since enthalpy is conserved and accounting for the heat release,

$$
H_{0_{2}}=H_{0_{1}}+\left(\chi_{2}-\chi_{1}\right) \frac{\theta_{c}}{\theta_{d}}
$$


Sanderson et al[23] give the remaining results that carry over directly;

$$
\begin{gathered}
K_{2}=K_{1}\left(\cos ^{2} \beta+\frac{\sin ^{2} \beta}{\hat{\rho}^{2}}\right) . \\
\hat{\rho}_{d_{2}}=\frac{\hat{\rho}_{d_{1}}}{\hat{\rho}} .
\end{gathered}
$$

Identity (13) then yields $P_{2}$ and the computation for the downstream shock wave proceeds as before using the above parameters. Recursive application of the above results yields the full solution for complex interaction problems.

\subsection{Kinetic Re-Normalization}

The normalization of the reaction rates downstream of a sequence of shock waves must be adjusted to common bases for comparison and these are chosen to be the characteristic reaction lengths for the free stream, $\ell_{i_{\infty}}$ and $\ell_{d_{\infty}}$, for the induction and dissociation rates respectively. At this point we adjust our notation slightly for multiple wave interaction problems. The subscript, $\infty$, refers to the free stream, and a numerical subscript identifies a shock wave and its corresponding downstream state.

For the $\mathrm{j}$-th wave that crosses a streamline in a complex interaction problem, we adjust the normalization back to the freestream state,

$$
\begin{aligned}
& \left.\hat{\Delta}_{j_{N}}\right|_{\infty}=\frac{\Delta_{j_{N}}}{\ell_{i_{\infty}}}=\frac{\Delta_{j_{N}}}{\ell_{i_{j_{-}}}} \frac{\ell_{i_{j_{-1}}}}{\ell_{i_{j_{-1}}}} \frac{\ell_{i_{j_{-1}}}}{\ell_{j_{\infty}}}=\frac{\Delta_{j_{N}}}{\ell_{i_{j_{-}}}} \frac{u_{j_{-1}}}{u_{j_{-1}}} \frac{u_{j_{-1}}}{u_{\infty}}\left(\frac{\rho_{\infty}}{\rho_{j}}\right)^{\mu} \\
& =\frac{\Delta_{j_{N}}}{\ell_{j_{k_{-}}}} \sin \beta_{1} \frac{u_{j_{-1}}}{u_{\infty_{N}}}\left(\frac{\rho_{\infty}}{\rho_{j}}\right)^{\mu}=\left.\hat{\Delta}_{j_{N}}\right|_{j_{-1}} \sin \beta_{1} \sqrt{\frac{K_{j_{-1}}}{K_{\infty_{N}}} \prod_{k=1}^{j_{-k}=1} \hat{\rho}_{j_{-k}}^{-\mu},}
\end{aligned}
$$

where the scaled induction length, $\left.\hat{\Delta}_{j_{N}}\right|_{\infty}$, is the normal distance from shock $j$ to the reaction zone downstream of it. Although the shock normal induction length is the most easily observed chemical reaction scale, note the equivalent scaled induction length in the streamline direction,

$$
\left.\hat{\Delta}_{j}\right|_{\infty}=\frac{\left.\hat{\Delta}_{j_{N}}\right|_{\infty}}{\sin \left(\beta_{j}-\delta_{j}\right)} .
$$

As before [23] the renormalized dissociation rate is similarly given by;

$$
\frac{d \alpha_{j}}{d \hat{x}_{\infty}}=\frac{d \alpha_{j}}{d \hat{x}_{j_{N}}} \sin \left(\beta_{j}+\sum_{k=1}^{j_{-k}=1} \delta_{j_{-k}}\right) \sqrt{\frac{K_{\infty}}{K_{j_{-1}}}} \prod_{k=1}^{j_{-k}=1} \hat{\rho}_{j_{-k}} .
$$


The first terms on the right-hand sides of equations (30) and (32), are given by equations (15) and (16) respectively using the normal components of the parameters for the $\mathrm{j}$-th shock. The trigonometric terms adjust for the appropriate vector components of velocity and the differing alignment of the $x_{\infty}$ and $x_{j}$ coordinates, the square root terms reflect the differing convective speeds, whereas the last product terms compensate for the scaling of the pre-exponential density dependence of the reaction rates. The notation, $i_{-j}$, refers to the sequence of upstream shocks involved in the normalization (e.g. for shock a $j=6$ with waves $j=5, j=3$ and $j=1$ upstream of it; $j_{-k} \in[5,3,1]$ with $j_{-1}=5, j_{-2}=3$ etc. $)$.

\subsection{Transverse Wave Structure Solution}

Represent the preceding model for the oblique detonation wave jump conditions and their mapping into the pressure-flow deflection angle $(p-\delta)$ plane by the notation,

$$
\left(\hat{\rho}_{2}, \hat{p}_{2}, \delta_{2}, P_{2}, H_{0_{2}}, \alpha_{2}, \chi_{2}\right)=\mathcal{J}\left(P_{1}, H_{0_{1}}, \alpha_{1}, \chi_{1}, \hat{\rho}_{d}, \beta_{1}\right) \text {. }
$$

Note that as before the reaction rates remain indeterminate and the solution is parameterized in terms of $\alpha$ and $\chi$. For a transverse wave system propagating at track angle, $\phi$, and at streamwise velocity ratio, $\Omega$, relative to the ideal one-dimensional Chapman-Jouguet conditions, $P_{C J}$ and $H_{0_{C J}}$, we have the dimensionless parameters;

$$
\begin{gathered}
P_{\infty}=\frac{P_{C J}}{\Omega^{2}\left(1+\tan ^{2} \phi\right)} \\
K_{\infty}=K_{C J} \Omega^{2}\left(1+\tan ^{2} \phi\right)
\end{gathered}
$$

such that

$$
H_{0_{\infty}}=K_{\infty}\left(1+2 P_{\infty} \frac{4+\alpha_{\infty}}{1+\alpha_{\infty}}\right)+\alpha_{\infty}
$$

and

$$
M_{\infty}=\sqrt{\frac{3}{\left(4+\alpha_{\infty}\right) P_{\infty}}}=M_{C J} \Omega \sqrt{1+\tan ^{2} \phi},
$$

where the subscript, $\infty$, now represents the free stream conditions in a frame of reference fixed with respect to and aligned with the flow direction relative 
to the transverse shock system. Applying the notation (33) to the proposed physical model (Figure 5) we obtain the system of equations,

$$
\begin{aligned}
\left(\hat{\rho}_{2}, \hat{p}_{2}, \delta_{2}, P_{2}, H_{0_{2}}, \alpha_{2}, \chi_{2}\right) & =\mathcal{J}_{R}\left(P_{\infty}, H_{0_{\infty}}, \alpha_{\infty}, \chi_{\infty}, \hat{\rho}_{d}, \beta_{2}\right), \\
\left(\hat{\rho}_{1}, \hat{p}_{1}, \delta_{1}, P_{1}, H_{0_{1}}, \alpha_{1}, \chi_{1}\right) & =\mathcal{J}_{F}\left(P_{\infty}, H_{0_{\infty}}, \alpha_{\infty}, \chi_{\infty}, \hat{\rho}_{d}, \beta_{1}\right), \\
\left(\hat{\rho}_{3}, \hat{p}_{3}, \delta_{3}, P_{3}, H_{0_{3}}, \alpha_{3}, \chi_{3}\right) & =\mathcal{J}_{F}\left(P_{1}, H_{0_{1}}, \alpha_{1}, \chi_{1}, \hat{\rho}_{d} / \hat{\rho}_{1}, \beta_{3}\right) .
\end{aligned}
$$

Note that here we have reverted to the subscripting scheme used for multiple shock wave interactions. Matching the pressure and flow deflection angle across the shear layer that originates at the primary $\lambda$-point that forms on the main detonation front we have,

$$
\begin{gathered}
\hat{p}_{2}=\hat{p}_{1} \hat{p}_{3}, \\
\delta_{2}=\delta_{1}+\delta_{3} .
\end{gathered}
$$

At the secondary $\lambda$-point formed at the junction of the reactant jet wave, 3 , and the transverse detonation Mach stem, 4, we have the additional equations,

$$
\begin{gathered}
\left(\hat{\rho}_{4}, \hat{p}_{4}, \delta_{4}, P_{4}, H_{0_{4}}, \alpha_{4}, \chi_{4}\right)=\mathcal{J}_{R}\left(P_{1}, H_{0_{1}}, \alpha_{1}, \chi_{1}, \hat{\rho}_{d} / \hat{\rho}_{1}, \beta_{4}\right) . \\
\left(\hat{\rho}_{5}, \hat{p}_{5}, \delta_{5}, P_{5}, H_{0_{5}}, \alpha_{5}, \chi_{5}\right)=\mathcal{J}_{F}\left(P_{3}, H_{0_{3}}, \alpha_{3}, \chi_{3}, \hat{\rho}_{d} /\left(\hat{\rho}_{1} \hat{\rho}_{3}\right), \beta_{5}\right) . \\
\hat{p}_{4}=\hat{p}_{3} \hat{p}_{5}, \\
\delta_{4}=\delta_{3}+\delta_{5} .
\end{gathered}
$$

We argue that the scale of the jet structure is determined by the relevant chemical scales local to the transverse wave system. so that the secondary triple point, $\lambda_{2}$, moves at the same velocity and in the same frame of reference as the primary triple point, $\lambda_{1}$. In this way we avoid reference frame modifications such as those posed by the Law-Glass model for double Machreflection of shock waves. Collectively, equations (34)-(46) represent a closed system of equations with one shock angle $\beta_{i}(i \in[1,5]), \phi, \Omega, P_{C J}, H_{0_{C J}}, \alpha_{\infty}$, $\chi_{\infty}$ and $\hat{\rho}_{d}$ as free parameters. Reflecting the assumed behavior of the jet shock system, equations (39), (40) and (44) are evaluated using chemically frozen thermodynamics with $\chi_{1,3,5}=\chi_{\infty}$ and $\alpha_{1,3,5}=\alpha_{\infty}$, whereas equations (38) and (43) are evaluated with the reactive models developed in section (3). The validity of the frozen approximation for shocks 1,3 and 5 will be validated by subsequent determination of the non-equilibrium reaction rates. 
As before (see Sanderson et al [9]) the important conclusion is that all of the wave angles at the two mutually inverted $\lambda$-points and hence the flow properties in the supersonic jet are completely determined up to the reaction length scales that remain indeterminate. Satisfaction of the conservation laws at any point along the main detonation front requires that it remain contiguous, with potentially finite curvature, or that it be punctuated by the shock interaction structure described above $[12,13]$, at some indeterminate length scale, and that the surrounding shock layer flow distort to accommodate the necessity of its existence. The undetermined length scales are derived from the interaction between the flow length scales and chemical length scales, $\ell_{i_{\infty}}=\frac{u_{\infty} A}{\rho_{\infty}^{\mu}}$ and $\ell_{d_{\infty}}=\frac{u_{\infty}}{C \rho_{\infty} \theta_{d}^{\eta}}$.

\section{Solution Parameterization and Closure}

In the case of hypervelocity shock interference with hyperbolic upstream influence, the local solution is completely determined by specification of the free stream conditions $\left(P_{\infty}, H_{0_{\infty}}, \alpha_{\infty}\right.$ and $\left.\hat{\rho}_{d}\right)$ along with specification of the incident shock angle, $\beta_{1}$ (note that the free stream reaction progress variable, $\chi_{\infty}$, and the reference frame parameters, $\phi$ and $\Omega$, are irrelevant in this case).

Reflecting the importance of downstream influence in the case of unsteady detonation propagation (note the limiting Chapman-Jouguet condition for 1D detonation and Hornung's information condition ${ }^{5}$ for Mach reflection (see Hornung et al $[16,25]$ and recent developments by Mouton \& Hornung $[28,29]))$ we close the current solution indirectly based on the downstream flow properties. Noting that the detonation front velocity fluctuates periodically about the mean value during each cellular cycle, we choose to study the temporally local condition whereby $\Omega=1$ relative to the apriori determinable $1 \mathrm{D}$ solution given by $P_{C J}, H_{0_{C J}}, \alpha_{\infty}, \chi_{\infty}$ and $\hat{\rho}_{d}$. Further noting the apparently free-running, self-sustaining transverse detonation stems observed in experimental detonations, we close the system of equations by imposing the requirement $\beta_{4}=\pi / 2$ with $M_{4_{N}}=1$. This requirement is consistent with a local interpretation of the CJ hypothesis in the immediate spatial and temporal frame of reference fixed with respect to the transverse

\footnotetext{
${ }^{5}$ Hornung argues that solution domains for the Mach reflection problem are dependent on the availability of an information path that supplies a length scale from the global flow field to the local point of shock intersection.
} 
wave ${ }^{6}$. Note that the sensitivity of flow deflection angle with respect to shock wave angle for nearly normal waves, along with the experimentally observed normality of the $\lambda_{2}$-shear layer with respect to the detonation stem, 4 , are strongly supportive of this approach. These requirements are sufficient to allow solution for the remaining wave angles, $\beta_{1}-\beta_{3}, \beta_{5}$ along with the transverse wave track angle $\phi$.

Over-driven solutions are also admitted, with all of $\Omega, M_{4_{N}}$ and $\beta_{4}$ as free parameters, with the CJ case representing the isolated solution that corresponds to the minimum admissible self-sustaining global and transverse wave velocities. The over-driven solutions are sensitive to locally induced variations in over-drive ratio, $\Omega$, and hence track angle, $\phi$. In the case of locally accelerated wave fronts, both throughout the cellular cycle and for spontaneously arising wavefront bifurcations, it should be possible to improve the fit based on local triple point speed and track angle data. In the absence of such data, all solutions shown are strictly based on the assumption of CJ propagation of the main front with $\Omega=1$ and $\phi$ determined by CJ propagation of the transverse detonation stem.

\section{Representative Solutions}

\subsection{Strongly Unstable Mixtures: $\mathrm{C}_{2} \mathrm{H}_{4}-3 \mathrm{O}_{2}-10.5 \mathrm{~N}_{2}$}

We begin by considering the solution for a representative strongly unstable mixture, $\mathrm{C}_{2} \mathrm{H}_{4}-3 \mathrm{O}_{2}-10.5 \mathrm{~N}_{2}$ that figures in the work of Austin [1]. Austin computes an ideal 1D Chapman-Jouguet velocity of $1844 \mathrm{~m} / \mathrm{s}$ for this mixture using the detail equilibrium thermo-chemical model STANJAN (Reynolds [30]) based on initial conditions of $287 \mathrm{~K}$ and $20 \mathrm{kPa}$. Computing the 1D Chapman-Jouguet velocity using the current model allows determination of the equivalent $\theta_{c}=6890 \mathrm{~K}$ for this mixture. Similarly Austin uses the detailed kinetic mechanisms of Konnov [31] and Warnatz [32] to calculate a reduced effective activation energy $\frac{\theta_{a}}{T}=\frac{\Theta_{\infty}}{\hat{T}} \frac{\theta_{a}}{\theta_{d}}=12.1$ evaluated at the post shock conditions (see equations (4) and (15)). Again matching to the frozen solution predicted by the current simple model, we obtain $\theta_{a}=20300 \mathrm{~K}$. The

\footnotetext{
${ }^{6}$ An alternative closure, $\beta_{1}=\pi / 2-\phi$, with $\phi$ obtained from soot foils is discussed in the literature. This yields a normal retreating main shock front in the lab frame, nominally consistent with experimental data, and is durable in the absence of a CJ solution as discussed above. This closure however has no formal basis and appears to overly restrict the forms of solution that can be obtained.
} 
remaining parameters for the IDG model governing the dissociation of the Nitrogen diluent are given in table 1. The solution shown in figure 6 then follows using the dimensionless forms and methodology described in sections 3.4 and 4 .

Figures 7 and 8 overlay Austin's Schlieren and OH PLIF images with the predicted wave angles and indicate correspondence with the observed shock and flow deflection angles. In both cases the predicted self propagating transverse detonation stem is apparent. The wave patterns has been nominally scaled to fit the observed images since the length scale remains indeterminate in the current local model. The OH PLIF images also reflect the relative induction lengths for the various shock waves as indicated in the solution shown in the caption of figure 6 . The absence of OH PLIF signal within the jet region formed between the two $\lambda$-points is obvious along with the prominent mixing regions originating from them, reflecting the predicted $150 \times$ increase in induction length for the jet fluid. The projected streamlines upstream from the two $\lambda$-points, shown as dotted lines, highlight the extensive free stream catchment area of the reduced entropy jet structure that is consistent with the extensive appearance of unreacted gas pockets in experimental visualizations and CFD predictions of strongly unstable detonations.

The retreating main detonation front is predicted to be essentially nonreactive with $\approx 10^{6} \times$ relative induction length, confirming the self-consistency of the freezing wave 1 in the formulation of the model (similar conclusions apply to waves 3 and 5). Indeed, irrespective of kinetic considerations, further examination reveals that shock 1 is under-driven relative to the CJ condition and as such there is no equilibrium reacted flow solution for this wave. Note also that since the downstream waves of the jet shock system, 3 and 5 , must produce additional entropy beyond that produced by the retreating main detonation front alone; then the induction length within the jet shock system must similarly be no greater than that in the region downstream of the retreating main detonation front itself. Careful consideration is therefore needed of the implications of the regions of OH PLIF signal that may be observed down-stream of the retreating main front in figures 7 and 8 .

In the case of figure 8 the above requirement for self consistency of the relative induction lengths appears to be satisfied, as may be observed by comparing the visually estimated length of the jet observed in the OH PLIF image with the somewhat longer distance observed from the secondary $\lambda$ point to the retreating detonation front extrapolated along the upstream particle path. Extending our interest from the particle path immediately 


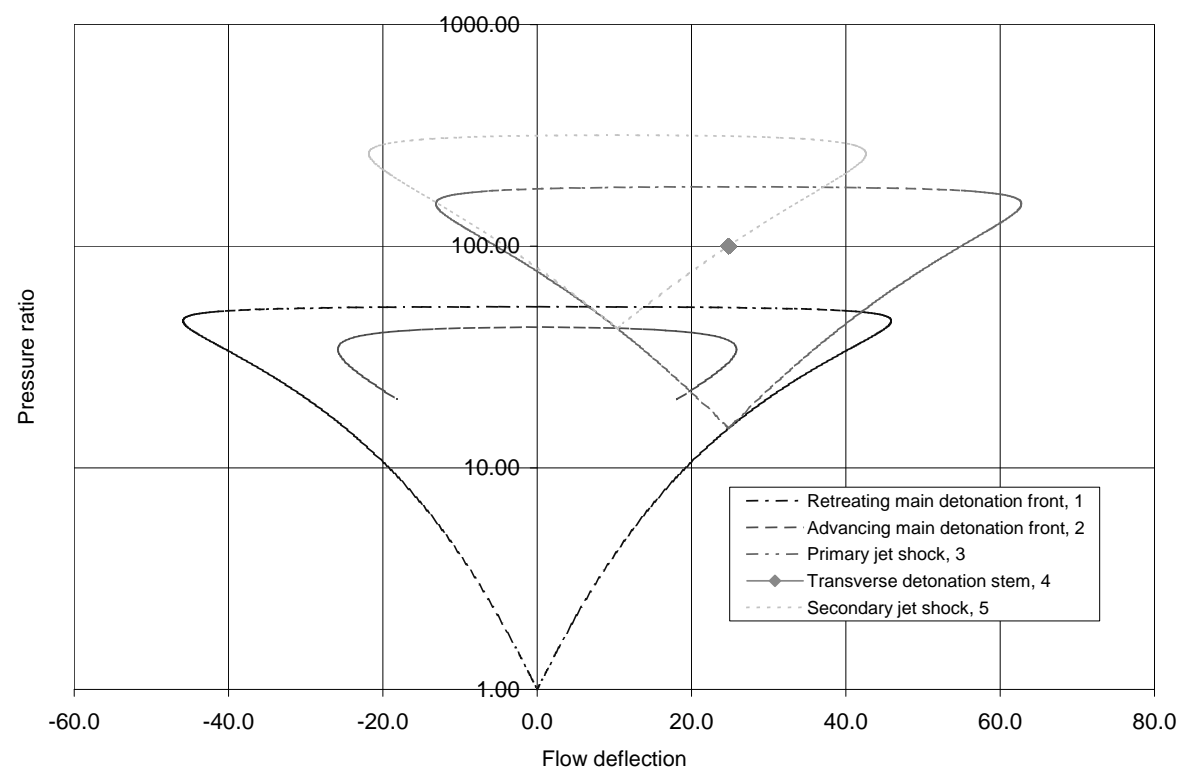

Figure 6: $p-\delta$ plane representation of transverse wave jet structure for the strongly unstable mixture $\mathrm{C}_{2} \mathrm{H}_{4}-3 \mathrm{O}_{2}-10.5 \mathrm{~N}_{2}$. The model parameters are; $P_{\infty_{C J}}=0.0251$ (or $M_{\infty_{C J}}=5.47$ ), $H_{0_{\infty_{C J}}}=0.0891, \Theta_{\infty}=394 ., \hat{\rho}_{d_{\infty}}=5.5 \times 10^{5}, \theta_{c} / \theta_{d}=0.0609, \theta_{a} / \theta_{c}=2.94$ (or $\left.\theta_{a} / \theta_{d}=0.179\right), \alpha_{\infty}=\chi_{\infty}=0, \eta=-2.5$ and $\mu=1$. The waves and their respective polars are identified in figure 5 and in the legend. Note that the transverse detonation stem polar collapses to a single point at the CJ state. Flow deflection angle, $\delta$, is referenced to the flow direction approaching the transverse wave system in a frame fixed with respect to the primary $\lambda$-point. The solution obtained is; $\phi=36.8^{\circ}, \beta_{1}=32.3^{\circ}, \beta_{2}=84.8^{\circ}$, $\beta_{3}=-29.3^{\circ}, \beta_{5}=35.0^{\circ}, \delta_{1}=24.8^{\circ}$ and $\delta_{2}=\delta_{1}+\delta_{3}=10.4^{\circ}$ such that $\beta_{4}=90^{\circ}$ with $\delta_{4}=\delta_{3}+\delta_{5}=0^{\circ}$ and $M_{4}=1$ at the CJ condition for the transverse detonation stem. The dimensionless induction length normal to the main advancing detonation front, $\left.\hat{\Delta}_{2_{N}}\right|_{\infty}=$ 55.6. The ratios of the induction lengths for the remaining waves are; $\frac{\left.\hat{\Delta}_{1_{N}}\right|_{\infty}}{\left.\hat{\Delta}_{2_{N}}\right|_{\infty}}=2.8 \times 10^{6}$, $\frac{\left.\hat{\Delta}_{3_{N}}\right|_{\infty}}{\left.\hat{\Delta}_{2_{N}}\right|_{\infty}}=7.8 \times 10^{3}, \frac{\left.\hat{\Delta}_{4_{N}}\right|_{\infty}}{\left.\hat{\Delta}_{2_{N}}\right|_{\infty}}=0.63$, and $\frac{\left.\hat{\Delta}_{5_{N}}\right|_{\infty}}{\left.\hat{\Delta}_{2_{N}}\right|_{\infty}}=154$. . At this level of dilution $\mathrm{N}_{2}$ dissociation levels remain negligibly low within the scope of the current model. Although $\mathrm{NO}$ and dissociated products will form, the evaluation of a reduced effective dissociation energy to capture the effect would be tangential to the direction of the current study.

upstream of the secondary $\lambda$-point to consider the induction zone behind the entire retreating detonation front, we observe a uniform induction length with only smooth variations that are plausibly explained by spatial non- 

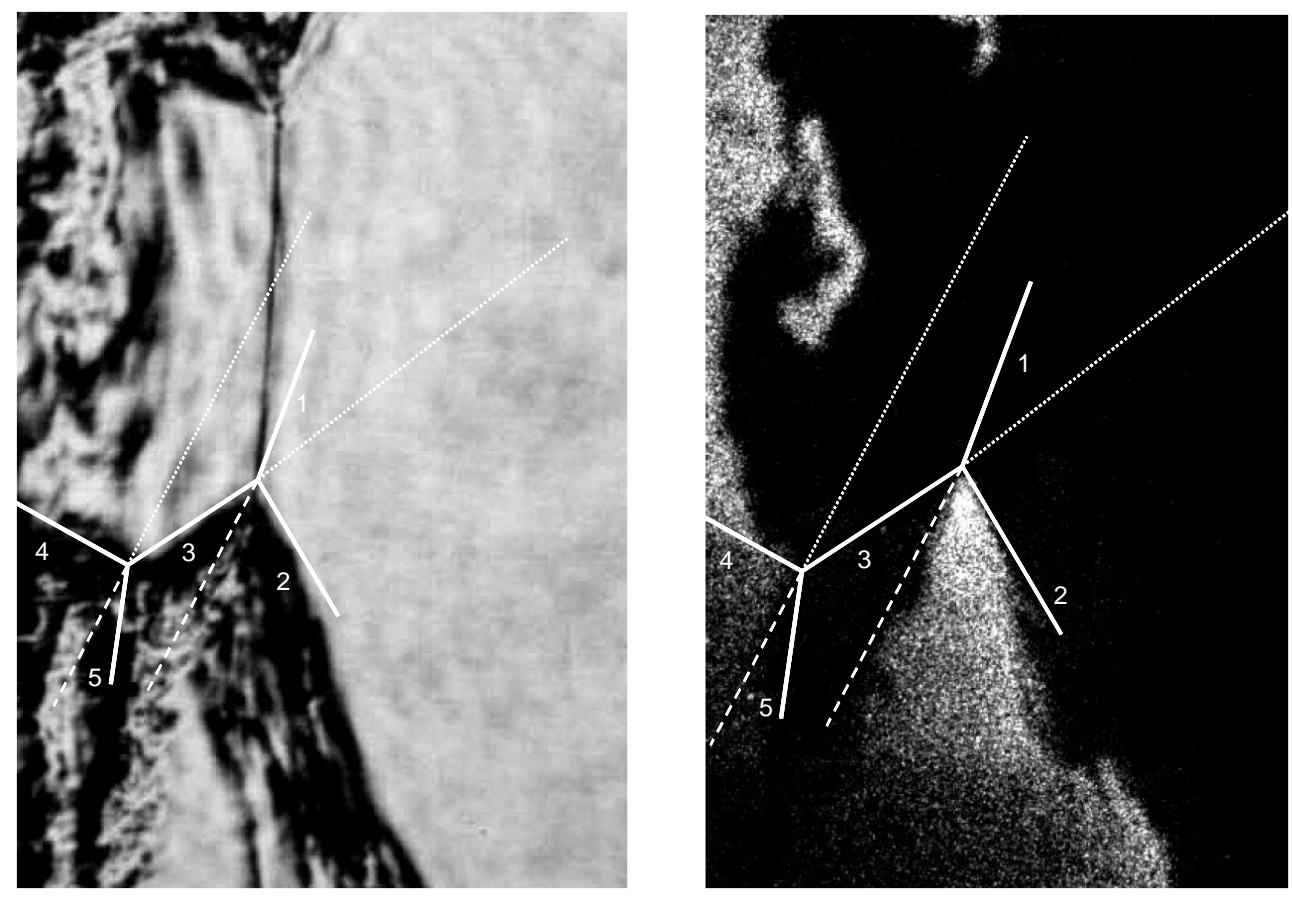

Figure 7: Comparison of predicted wave angles with Schlieren (left) and OH PLIF (right) images for a strongly unstable mixture of $\mathrm{C}_{2} \mathrm{H}_{4}-3 \mathrm{O}_{2}-10.5 \mathrm{~N}_{2}$ at an initial pressure of $20 \mathrm{kPa}$ (Austin [1], figure 5.7). Physical height of the displayed image is $30 \mathrm{~mm}$. Solid lines are the transverse jet structure shock waves local to the $\lambda$-points. Dashed lines are the shear layers downstream from the $\lambda$-points. Dotted lines are the upstream streamlines that pass through the $\lambda$-points and indicate the region of incoming flow that passes through the reduced entropy rise jet shock system comprising waves 1,3 and 5. Model parameters and local solution as for figure 6 . Since the solution requires that the transverse detonation stem propagate at the local CJ condition, $\beta_{4}=90^{\circ}$ with $\delta_{4}=\delta_{3}+\delta_{5}=0^{\circ}$ and $M_{4}=1$, the streamline passing through the secondary $\lambda$-point is undeflected. Note the counter propagating system just entering the field of view at the top of the image for this example obtained late in the cellular cycle shortly prior to collision of the transverse wave systems.

uniformity (driven by shock curvature away from the immediate vicinity of the $\lambda$-points) and temporal unsteadiness (driven by local shock acceleration and decay throughout the cellular cycle) that are not captured in the current local model.

The interpretation for figure 7 is less clear however since the jet induction length extends beyond the field of view in this case. At a minimum, taking the limit imposed by the field of view as a lower bound on the length of the jet and 

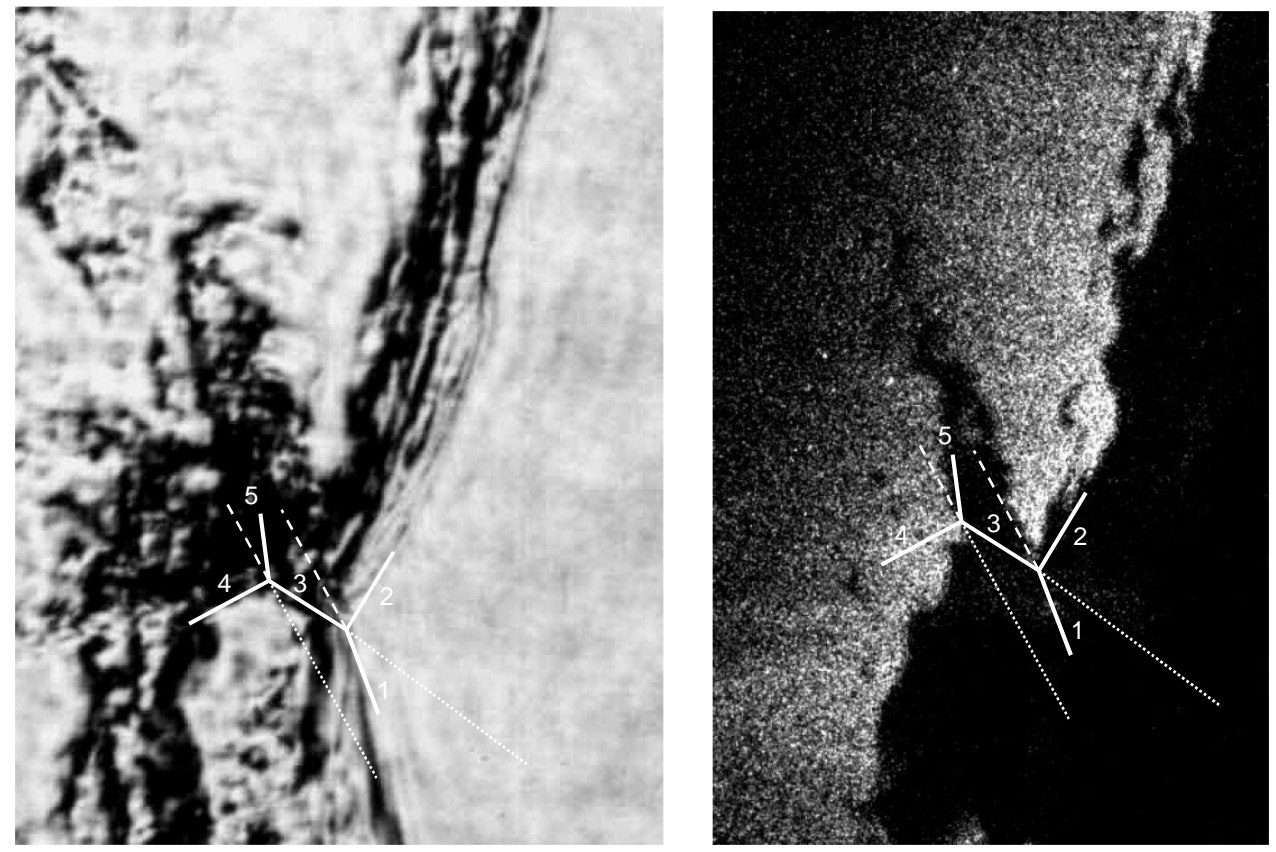

Figure 8: Comparison of predicted wave angles with Schlieren (left) and OH PLIF (right) images for a strongly unstable mixture of $\mathrm{C}_{2} \mathrm{H}_{4}-3 \mathrm{O}_{2}-10.5 \mathrm{~N}_{2}$ at an initial pressure of $20 \mathrm{kPa}$ (Austin [1], figure 7.2). Legend, dimensions and local solution as for figure 7.

the induction length extrapolated upstream along the particle path from the secondary $\lambda$-point, we can conclude that there is no immediate contradiction of the model. Extending the argument away from the immediate vicinity of the secondary $\lambda$-point is more difficult however. In contrast to the spatial regularity of the retreating front induction zone observed in figure 8 , we observe a large fragmented region of $\mathrm{OH}$ PLIF signal in the corresponding region of figure 7. Again extrapolating along the deflected particle path (not shown) that would lead from this $\mathrm{OH}$ fragment to the supposed beginning of induction, we find that (given the expected induction length based on that observed at secondary $\lambda$-point) the induction zone extends both beyond the field of view and into a region that has been consumed by an additional counter-propagating transverse wave that has just entered the top of the field of view. Furthermore, to fully understand the flow history along the particle path that led to this $\mathrm{OH}$ fragment, we are also required to extrapolate upstream in time. It is not possible to do this with certainty based on a 


\begin{tabular}{|l||}
$\theta_{d}=113200 \mathrm{~K}$ \\
$\rho_{d}=130000 \mathrm{~kg} \mathrm{~m}^{-3}$ \\
$m=14.0 \times 10^{-3} / 6.023 \times 10^{23} \mathrm{~kg}$ \\
$C=2.7 \times 10^{21} \mathrm{~m}^{3} \mathrm{~kg}^{-1} \mathrm{~s}^{-1} \mathrm{~K}^{2.5}$ \\
$\eta=-2.5$
\end{tabular}

Table 1: Constants for IDG model of $N_{2}$ diluent.

single image in the case of strongly unstable detonation whereby the prior history of transverse waves that might have passed through the region is highly irregular. As before, we simply conclude that there is no immediate contradiction, and recognize that additional positive conclusions necessarily require visualizations (likely CFD based) that resolve both the spatial and temporal development of the induction zone.

Formally, our local analysis relies on a spatially and temporally uniform frame of reference in the immediate vicinity of each individual transverse wave jet system. It is unsurprising that in the above examples we see indications of the limitations of local analysis whereby the various local reference frames and the global scales overlap.

\subsection{Comparison with CFD Predictions}

Figures 7 and 8 represent manifestations of the reactant jetting phenomena at different length scales, attributable to the self-sustaining nature of the CJ transverse detonation stem, originating in spontaneously occurring instabilities that occur at random locations along the shock front, and that are not reliant on an external mechanism of wave driven feedback for propagation. This aspect of the strong detonation instability and further validation of the model are apparent in the CFD predictions of Liang et al [17] whereby the instability appears at widely differing scales along the shock front, all exhibiting the same fundamental jet structure (see figure 9). The jet complex takes many forms representing a wide range of interactions of local and global scales. We observe highly compacted (upper overlay and insets, figure 9), jets bordering prominent self-propagating transverse detonation stems (see figure 10, right), through fully expanded jet structures with no apparent Mach stem but rather a prominent oblique jet shock and associated weak wave interactions generated by disturbances propagating from the downstream far field (lower overlay, figure 9). 


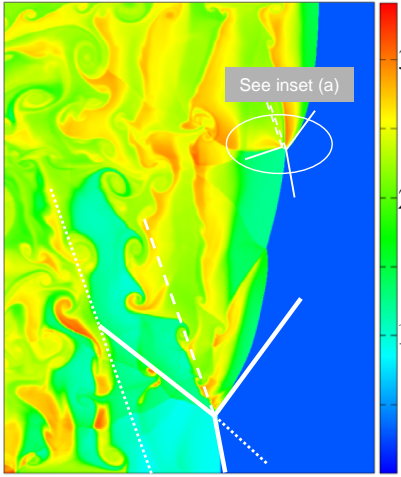

(a)

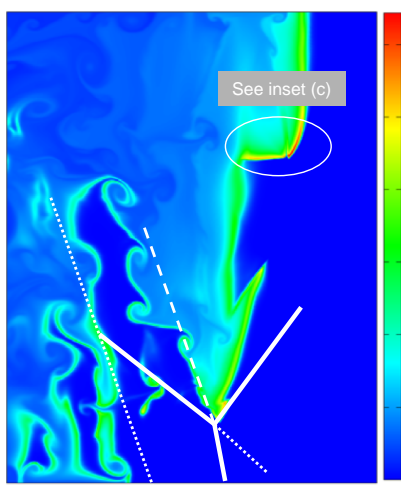

(c)

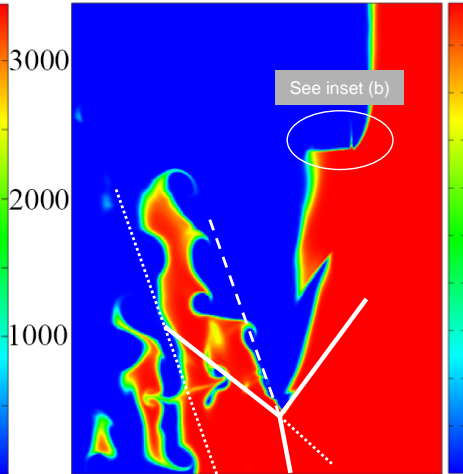

(b)

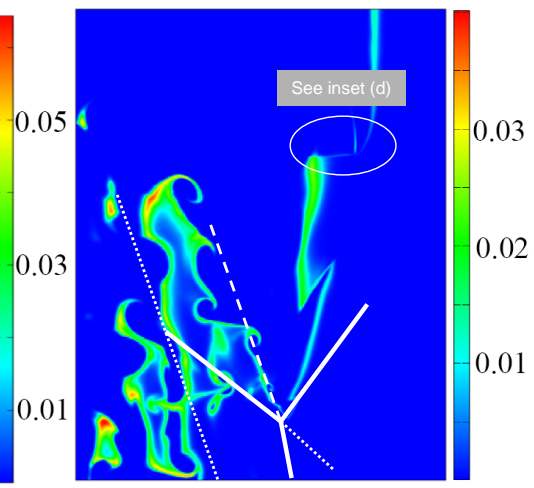

(d)

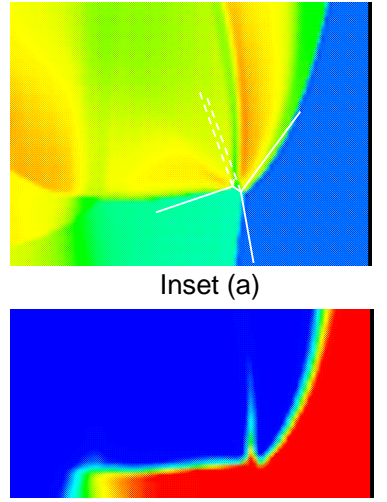

Inset (b)

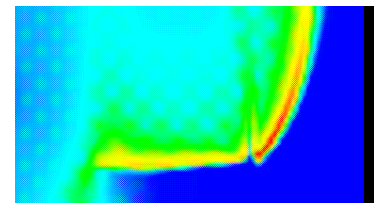

Inset (c)

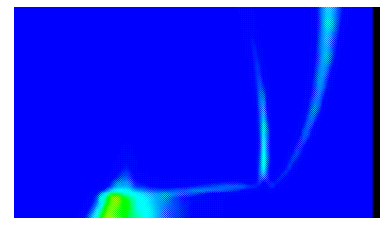

Inset (d)

Figure 9: Computed reaction zone structure and kinetic pathways for strongly unstable detonation (Liang et al [17]). Sub-plots are (a) temperature, (b) reactant pseudo-species mass fraction, (c) radical pseudo-species mass fraction, (d) intermediate pseudo-species mass fraction. The model parameters used to calculate the wave angles and local solution are; $P_{\infty_{C J}}=0.0406$ (or $M_{\infty_{C J}}=4.30$ ), $H_{0_{\infty_{C J}}}=0.0432, \Theta_{\infty}=377 ., \hat{\rho}_{d_{\infty}}=1.1 \times 10^{5}$, $\theta_{c} / \theta_{d}=0.0377, \theta_{a} / \theta_{c}=2.04$ (or $\left.\theta_{a} / \theta_{d}=0.0768\right), \alpha_{\infty}=\chi_{\infty}=0, \eta=-2.5$ and $\mu=1$. The solution obtained is; $\phi=42.6^{\circ}, \beta_{1}=37.3^{\circ}, \beta_{2}=83.4^{\circ}, \beta_{3}=-32.5^{\circ}, \beta_{5}=39.4^{\circ}$, $\delta_{1}=28.1^{\circ}$ and $\delta_{2}=\delta_{1}+\delta_{3}=14.8^{\circ}$ such that $\beta_{4}=90^{\circ}$ with $\delta_{4}=\delta_{3}+\delta_{5}=0^{\circ}$ and $M_{4}=1$ at the CJ condition for the transverse detonation stem. Flow deflection angle, $\delta$, is referenced to the flow direction approaching the transverse wave system in a frame fixed with respect to the primary $\lambda$-point. The dimensionless induction length normal to the main advancing detonation front, $\left.\hat{\Delta}_{2_{N}}\right|_{\infty}=1.53$. The ratios of the induction lengths for the remaining waves are; $\frac{\left.\hat{\Delta}_{1_{N}}\right|_{\infty}}{\left.\hat{\Delta}_{2_{N}}\right|_{\infty}}=282 ., \frac{\left.\hat{\Delta}_{3_{N}}\right|_{\infty}}{\left.\hat{\Delta}_{2_{N}}\right|_{\infty}}=41 ., \frac{\left.\hat{\Delta}_{4_{N}}\right|_{\infty}}{\left.\hat{\Delta}_{2_{N}}\right|_{\infty}}=0.67$, and $\frac{\left.\hat{\Delta}_{5_{N}}\right|_{\infty}}{\left.\hat{\Delta}_{2_{N}}\right|_{\infty}}=7.5$.

Liang also produced computational soot foil simulations that allow val- 

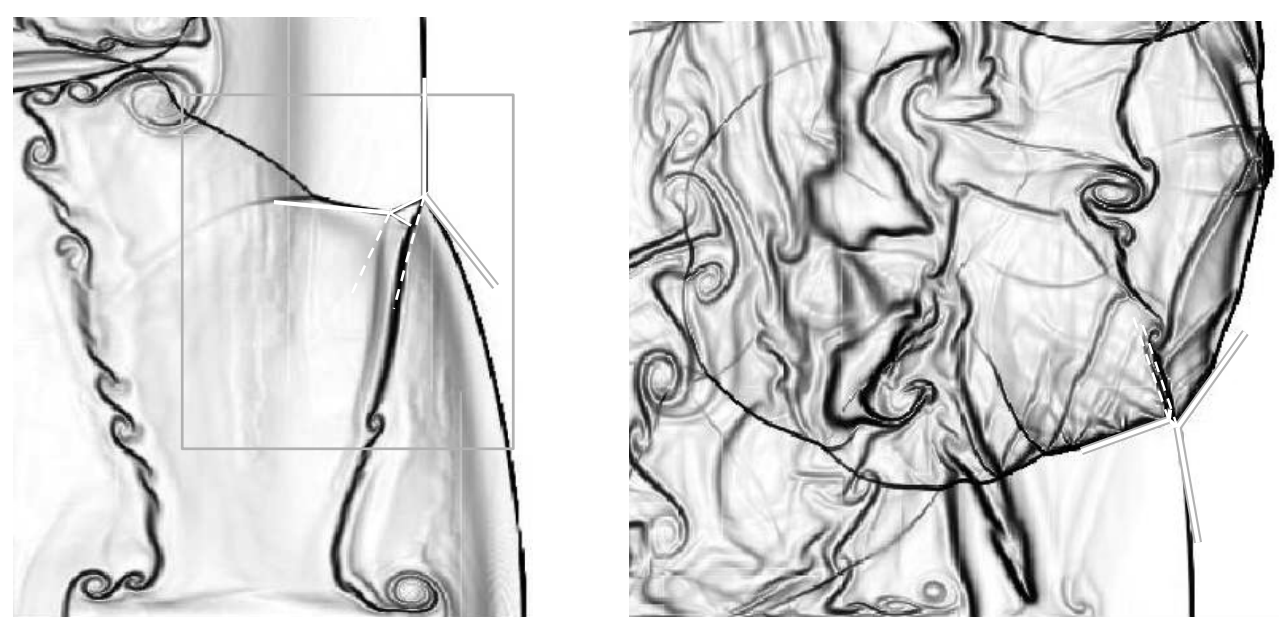

Figure 10: Computational Schlieren image of 2D weakly unstable (left) and strongly unstable (right) detonations. (Liang et al [17]). The boxed region for the weak case is magnified in figure 4 .

The model parameters used to calculate the wave angles and local solution for the weak case are; $P_{\infty_{C J}}=0.0196\left(\right.$ or $\left.M_{\infty_{C J}}=6.19\right), H_{0_{\infty_{C J}}}=1.1485$ (note that the total enthalpy includes the dissociation energy associated with limit $\alpha_{\infty} \rightarrow 1$ used to approximate a mono-atomic diluent in this contrived application of the IDG model), $\Theta_{\infty}=377 ., \hat{\rho}_{d_{\infty}}=8.0 \times 10^{4}, \theta_{c} / \theta_{d}=0.0714, \theta_{a} / \theta_{c}=1.66\left(\right.$ or $\left.\theta_{a} / \theta_{d}=0.119\right)$, $\alpha_{\infty}=\alpha_{1}=\alpha_{2}=\alpha_{3}=\alpha_{4}=\alpha_{5}=1$ (frozen dissociation chemistry to prevent the appearance of unwanted recombination shocks), $\chi_{\infty}=0, \eta=-2.5$ and $\mu=1$. The solution obtained is; $\phi=45.3^{\circ}, \beta_{1}=44.7^{\circ}, \beta_{2}=83.0^{\circ}, \beta_{3}=-48.4^{\circ}, \beta_{4}=-79.7^{\circ}, \beta_{5}=86.5^{\circ}$, $\delta_{1}=29.5^{\circ}, \delta_{2}=\delta_{1}+\delta_{3}=18.0^{\circ}$ and $\delta_{4}=\delta_{3}+\delta_{5}=-10.0^{\circ}$. Flow deflection angle, $\delta$, is referenced to the flow direction approaching the transverse wave system in a frame fixed with respect to the primary $\lambda$-point. The dimensionless induction length normal to the main advancing detonation front, $\left.\hat{\Delta}_{2_{N}}\right|_{\infty}=0.457$. The ratios of the induction lengths for the remaining waves are; $\frac{\left.\hat{\Delta}_{1_{N}}\right|_{\infty}}{\left.\hat{\Delta}_{2_{N}}\right|_{\infty}}=8.4, \frac{\left.\hat{\Delta}_{3_{N}}\right|_{\infty}}{\left.\hat{\Delta}_{2_{N}}\right|_{\infty}}=3.7, \frac{\left.\hat{\Delta}_{4_{N}}\right|_{\infty}}{\left.\hat{\Delta}_{2_{N}}\right|_{\infty}}=1.2$, and $\frac{\left.\hat{\Delta}_{5_{N}}\right|_{\infty}}{\left.\hat{\Delta}_{2_{N}}\right|_{\infty}}=1.5$. Model parameters and local solution for the strong case as for figure 9.

idation of the track angles predicted by the current model. Proceeding by the same method described in sections 3.4 and 4 , but for various values of over-drive parameter, $\Omega \in[0.85,1,1.15,1.3]$, we are able to compare the predictions with a representative detonation cell boundary (see figure 11). The correct trend of increasing track angle with reduced over-drive is reproduced, and the cyclical variation in main front velocity inferred by matching the predicted track angle to the observed cell boundary is consistent with 
computational and experimental results that show a typical variation in the range $1.3>\Omega>0.85$.

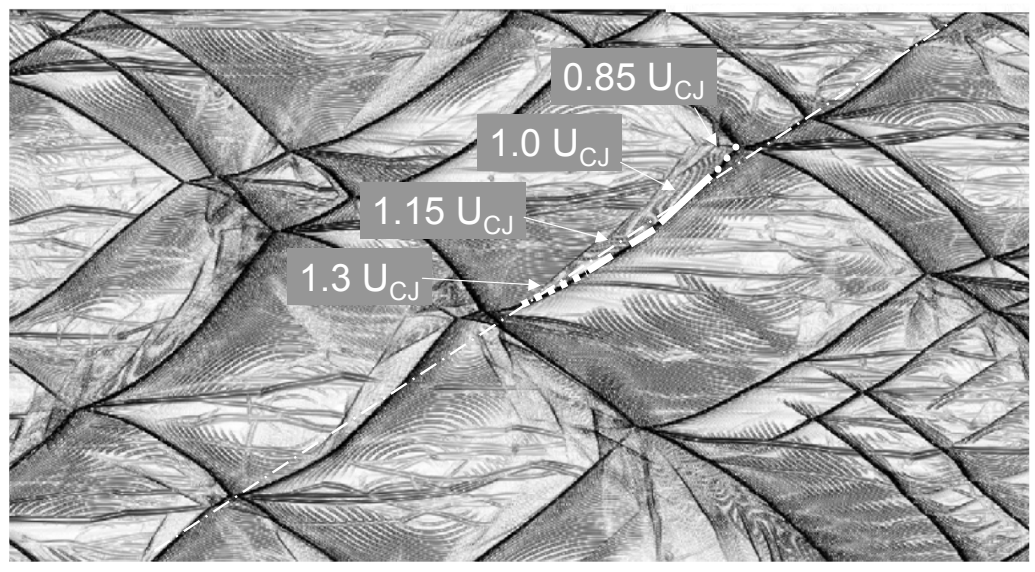

Figure 11: Computational soot foil for strongly unstable detonation (Liang et al [17]). Predicted track angles for $\Omega \in[0.85,1,1.15,1.3]$ yielding $\phi \in\left[51.1^{\circ}, 42.7^{\circ}, 32.5^{\circ}, 25.7^{\circ}\right]$ based on the hypothesis of normal CJ propagation of the transverse detonation stem are overlaid on a representative detonation cell boundary. Since this is a prediction based on a local model, the location of each predicted track angle is aligned based on its tangency with the observed track angle to demonstrate self consistency and does not connote an independent prediction of the spatial variation of $\Omega$ throughout the cell cycle. Note also that the mean track angle, denoted by the thin —- - line is not aligned with the segment for $\Omega=1$. This is due to the non-linear decay of the main detonation front velocity throughout the cellular cycle such that the average track angle $\left(39 .^{\circ}\right)$ does not coincide with the track angle $\left(43 .^{\circ}\right)$ at the average speed $(\Omega=1)$. Mixture as for figures 9 and 10 (strong case).

\subsection{Mono-Atomic Diluents: $\alpha_{\infty} \rightarrow 1$}

A full discussion of the solutions admitted by this formulation lies beyond the scope of this paper. We note however, that predictions of the model with values of $\alpha_{\infty} \rightarrow 1$, indicative of a mono-atomic diluent with $\gamma=5 / 3$, reveal a structural failure of the transverse CJ detonation stem solution discussed in section 5.1 whereby the transverse wave 4 is under-driven. In the absence of a reactive solution we seek a chemically frozen solution for all waves in the jet structure. Alternate solutions may however be possible early in the cellular cycle, with sufficient over-drive. 
We illustrate the key features of weak solutions by analyzing the weak case investigated by Liang (see figure 10). Equilibrium considerations alone (under-driven relative to CJ) require that waves 1 and 4 be treated as frozen in the vicinity of the jet, whereas kinetic considerations require that the main detonation front, 2 , must similarly be treated as frozen for a self consistent approximation of the chemical scales. As before, the reaction rates downstream of each of the jet system shock waves, 1,3 and 5, are suppressed but the effect is significantly less pronounced and almost absent downstream of wave 5 (normalized reaction lengths are noted in the caption of figure 10). From the consistency of the predicted induction lengths, and the absence of equilibrium solutions for waves 1 and 4, we see however that the assumption of frozen behavior downstream of all shocks in the vicinity of the interaction is kinetically self consistent in this case. The jet structure therefore takes the form of a nonlinear acoustically driven instability with the oblique downstream transverse wave driving the interaction. In the absence of a CJ condition to close the solution, predictions for a specific flow requires knowledge of the external flow field - here we match the solution based on the observed shock wave and flow deflection angle behind the main detonation front, 2 , since the deflection angles behind strong waves are the most sensitive, easily observable data. The predicted wave angles match well with the weakly unstable case investigated by Liang (see figure 10). The absence of turbulent or secondary wave structure in experimental observations of weakly unstable detonations is also consistent with this frozen treatment of the shock structure.

The effects noted above, and the implication of weak instability with reduced tendency to form unreacted gas pockets, apply generally to all systems with substantial mono-atomic dilution. This is consistent with the widely observed behavior of Argon diluted mixtures (e.g. Figure 1) and the limited data available for Helium diluted mixtures ${ }^{7}$. The results of section 5.1 demonstrate the behavior for strongly unstable diatomic mixtures. By extension we expect that polyatomic diluents will promote still stronger reactant jetting effects, and this is consistent with the data obtained by Austin [1] for $\mathrm{CO}_{2}$ based mixtures. The effect directly parallels that observed in the hypervelocity shock interference heating problem, whereby higher free stream Mach numbers and reduced ratio of specific heats, $\gamma$, significantly reduce

\footnotetext{
${ }^{7}$ Pintgen, F. Private communication.
} 
the entropy rise along the stagnation streamline that passes through the jet structure, thereby increasing the density at the stagnation point, reducing the temperature and greatly suppressing non-equilibrium dissociation in the jet fluid.

Despite the absence of a locally CJ detonation solution compatible with the requirements of equilibrium thermodynamics, the simple kinetics models used here indicate that the elongated induction zones predicted within the jet shock structure are significantly less pronounced as $\alpha_{1}$ increases (see the relative induction lengths noted in figure 10).

\section{Discussion}

\subsection{Impact of Jetting on Reaction Pathways}

Much recent literature has focused on the role of chemical kinetics in detonation instability, with the instability feedback mechanisms in both single and multi-dimensional cases being strongly dependent on the specifics of the kinetic model. Commonly, the influence of mixture kinetics is expressed via two quantities derived from the mechanism; the reduced effective activa-

tion energy $\left(\frac{\theta_{a}}{T}\right.$ here) and the ratio of induction time to energy release time scales (infinite in the current approximation). Larger values of both parameters are thought to be associated with stronger instability (see Austin [1]). Note that there is a fundamental synergy between these two parameters and the reduced entropy shock structure described here. The effects of a high velocity jet convecting mixtures with long induction times, along with the multiplicative impact of elevated reduced effective activation energies and temperature suppression in the reduced entropy shock structure and finally the exponential form of the Arrhenius reaction rate expression, combine to modulate massive changes in the kinetic state of the jet gas. The combined effect is consistent with observations of a highly fragmented flow field with widely disparate chemical and fluid mechanical scales and competing reaction pathways.

The mixtures studied by Liang were based on a hypothetical pseudomechanism designed to illustrate the impact of competing reaction pathways on detonation instability. Although it is not possible to directly relate these models to specific real mixtures, the weakly unstable case is representative of the behavior associated with Argon diluted mixtures whereas the strongly unstable case (note that a third strongly unstable mixture is not discussed here) exhibits characteristics expected to be important in H2-air mixtures by 
mimicking the behavior of peroxide species that are important to low temperature behavior. The 5-step model comprised a reactant species (Figure 9(b)), a radical species (Figure 9(c)), an intermediate species (Figure 9(d)), products and a non-reacting third body species reacting via radical production, chain branching, chain termination and recombination sub-reactions. Subreaction parameters were chosen to simulate the competition between chain branching and chain termination pathways that characterizes the extended second explosion limit ${ }^{8}$. Regions of low temperature and reduced total pressure loss in the jet favor chain termination whereas the high temperature and increased total pressure loss across the main shock fronts favor the chain branching pathway. This is consistent with the species plots of Liang that show the greatest radical concentrations formed behind the advancing main detonation fronts where they curve to accommodate the local jet solution (Figure 9(c) and inset) thereby aligning themselves more normally with respect to the direction of propagation of the triple point. Conversely, the highest concentrations of intermediate species formed by chain termination appear only at a significant distance downstream in regions of jet fluid that have been processed through the reduced entropy rise shock structures (Figure $9(\mathrm{~d})$ and inset). Beyond the above noted locations of the peaks of radical and intermediate concentration, these concentration fields are generally observed to be spatially complementary in the post shock flow-field, with the regions of overlap invariably coinciding with the shear layers generated at the shock wave $\lambda$-points.

Re-examining Liang's results we again note a fundamental synergy with the jetting mechanism. The shock structure provides a low temperature pathway for production and jetting of intermediate species that are important to low temperature detonation chemistry. It is apparent that for certain mixtures and initial conditions, jetting can produce a strongly bimodal distribution of jet processed and main shock processed fluid with independent reaction pathways and hence disparate detonative characteristics.

\footnotetext{
${ }^{8}$ Since such a model by design produces variable reduced effective activation energy, $\frac{\theta_{a}}{T}$, for different reaction pathways, we choose equivalent parameters for our current one-step model based on the CJ state. Regardless of this approximation, equilibrium considerations alone yield important conclusions when considering the current model relative to Liang's results.
} 


\subsection{Behavior of the Transverse Waves}

Formulation of the jet model described above causes us to re-examine prior experimental and computational visualizations to understand the embedded shock structure. This leads to several observations regarding the characteristics of the transverse waves that are indicative of important aspects of the physical behavior. There is a striking difference between the far field transverse waves observed in the weakly unstable case (Figure 10(a)) and in the strongly unstable case (Figure 10(b)) ${ }^{9}$. In the weak case (see also figure $1(\mathrm{a})(\mathrm{b})$ ) the far field wave forms as a wedge shaped envelope of circularly expanding downstream disturbances, the most important being the residual spherical blast waves from the line of preceding cell apexes, that ultimately intersects with and leads the transverse wave structure across the flow field. In the strongly unstable case (see also figure $1(\mathrm{~d})$ ) the far field transverse waves essential disappear. In intermediate cases a transitional behavior is apparent (see figure 1(c) and discussion below) whereby the far field transverse waves appear insipid and poorly formed.

The generic transverse wave consists of multiple components with disparate characteristics (see figure 5): the far-field transverse wave driven by pressure difference across the cell boundaries that adjoining advancing and retreating detonation half cells, the near field transverse wave generated directly at the primary triple point by the shock bifurcation, and the transverse stem that adjoins the near and far fields that is detonative in the case of strong instability. Indeed in the weak case the far-field and near-field transverse waves are of opposite families, highlighting their distinct identity, as may be inferred from the propagation of the far-field wave towards the main front in a frame fixed and oriented with respect to the primary triple point, and by appearance of a reflected wave where it joins with the downstream end of the Mach stem (see figure 10(a)). It is apparent therefore that in the weak case the far field transverse wave carries feedback from the far field to the detail transverse wave structure with the influence propagating along the hyperbolic characteristics that are incident on the near field region.

Contrast this with the behavior in the strong case (Figure 10(b)) whereby

\footnotetext{
${ }^{9}$ These computational images have been chosen since they clearly illustrate the phenomena. Equivalent images may be observed that lie between the two extremes of physical behavior illustrated here. The behavior is also observed in experimental images however the delineation of the role of the composite components of the transverse wave is obscured by the difficulty of experimental visualization.
} 
the far-field transverse wave is of the same family as the near field wave and originates from the downstream end of the self propagating transverse detonation stem where it presumably encounters the downstream extent of unreacted fluid. The far field transverse wave propagates into the surrounding pre-reacted fluid with the acceleration of the gas across the spreading wave balancing the over-pressure produced by the transverse detonation stem. The self propagating nature of the strong case, independent of a feedback mechanism from the far field is highlighted. Indeed in experimental visualizations of strongly unstable mixtures, the far-field transverse waves are observed to essentially disappear. It is possible that this disappearance is attributable to reduced optical sensitivity to the less coherent spherical wave fronts in comparison to strongly formed planar oblique wave in the weakly unstable case, however the CFD results are independent of this effect and illustrate the same behavior.

The strong case of Liang is particularly interesting since it illustrates a detonation exhibiting a spectrum of transitional behavior between the two extremes of weak and strong transverse wave behavior (see the boxed features in figure 12). Case 1 shows the formation of an oblique transverse far field wave, typical of that observed in weakly unstable mixtures, with the far field leading the near field across the flow. Case 4 shows the extreme opposite with a strongly formed transverse detonation stem, expanded to the width of the unreacted fluid behind the retreating main front, with a trailing oblique wave that transitions into a trailing expanding spherical wave in this presumably locally transient realization. Cases 2 and 3 however illustrate a competition in the information path that leads to the near-field jet system. In both examples there is a well formed transverse stem, presumably at the CJ state with sonic flow downstream. The transverse waves beyond the end of the stems however, barely keep pace with the progress of the detonation stem, appearing nearly horizontal with a lumpy, incoherent appearance and propagating at close to sonic speed (since the wave is weak) through the pre-reacted fluid downstream from the end of the stem.

These examples, consistent with numerous experimental visualizations of the same phenomena (see Austin [1]), appear to reveal a shift in the feedback mechanism between the near and far field flows that is associated with the transition from weakly to strongly unstable detonation. The mechanism appears to be associated firstly with thermodynamic considerations that allow the existence of a self sustaining transverse detonation stem, and secondly with the subsequent competition between feedback mechanisms due to propa- 


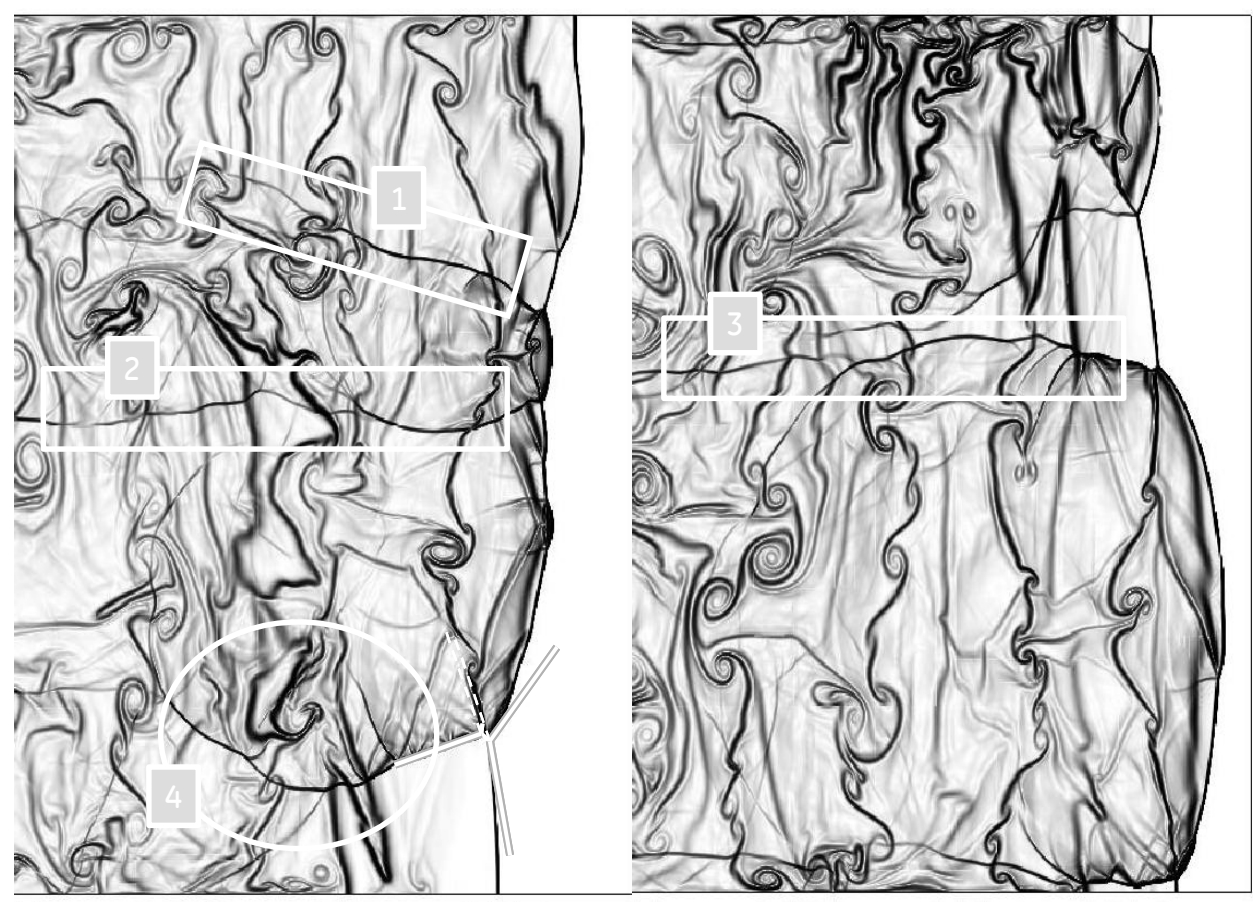

Figure 12: Computational Schlieren image of transitional behavior of the far-field transverse waves in strongly unstable detonation. (Liang et al [17]). Various behaviors of the far-field transverse wave are apparent: Case 1 - oblique incident wave leading the near field interaction; Cases 2, 3 - transitional; Case 4 - self propagation of near field structure. Model parameters and local solution as for figure 9.

gation of influence through the reacted and unreacted regions of downstream flow (note again the significance of Hornung's information condition in the study of transition in Mach reflection). There appears to be a range of overlap between these primary and secondary mechanisms, perhaps consistent with the appearance of soot foils in the case of strongly unstable detonation which exhibit a persistent large scale cellular structure overlaid with an irregular smaller scale substructure.

\subsection{Reactant Jet Curvature}

Driven by the need to satisfy the local requirements of shock bifurcation along the main detonation front, we see strong distortions of the bulk flow 
features to accommodate the necessity of the local existence of the jet shock structure. Perhaps counter-intuitively, this requirement holds irrespective of the local scale of the jet structure. Indeed small realizations of the jet structure drive increasingly strong distortions of the surrounding flow (see for example the inset (a) of figure 9).

This phenomenon is easiest to understand by first examining the behavior in the hypervelocity shock interaction flow field where equivalently strong curvature of the supersonic impinging jet is observed. Figure 3 shows the most important case whereby the stagnation streamline on the blunt body passes directly through the reduced entropy jet shock system. As the impingement point is raised with respect to the body, the jet narrows and increasingly curves upwards, to the point that the supersonic jet curves and passes above the body, with the stagnation streamline now crossing the extended bow shock, 4, below the interaction zone. As noted, given only the free stream conditions, and the impinging shock wave angle $\beta_{1}$, all wave angles in the supersonic jet structure and the thermodynamic state of the gas in it are thereby determined. Thus the strength of wave 5 , that determines the pressure difference across the jet is fixed. It follows directly that the jet curvature is therefore inversely proportional to its width since streamline curvature is proportional to the transverse pressure gradient, approximated by the jet pressure difference divided by its width. The secondary jet wave, 5 , then propagates along the supersonic jet by free streamline reflection from the surrounding subsonic shock layer, providing the mechanism that induces the curvature noted above.

Reactant jetting in unstable detonation produces a parallel phenomenon; the jet shock structure is again predetermined by the mixture and by satisfaction of the CJ condition both globally to determine the mean propagation and locally to determine the track angle and propagation of the transverse detonation stem. All wave angles and thermodynamic states are fixed and, as before, compressing the scale of the interaction due to the influence of the external flow drives increasingly strong curvature of the jet and surrounding features. For the cases considered in the current paper, the effect is somewhat less pronounced for than in the hypervelocity flow case, as can be understood from the Mach numbers and waves angles that are produced.

The strongly unstable $\mathrm{C}_{2} \mathrm{H}_{4}-3 \mathrm{O}_{2}-10.5 \mathrm{~N}_{2}$ mixture studied in section 5.1 produces $M_{5}=2.0$ downstream of the secondary jet shock, 5 . The strongly unstable CFD results of section 5.2 correspond to $M_{5}=1.7$. Both produce moderately distorted flow features. The weakly unstable case of section 5.3 
with mono-atomic diluent produces a subsonic jet beyond the immediate vicinity of the triple points with $M_{5}=0.8$. The free-streamline supersonic reflection of the secondary jet shock therefore fails, the pressure gradient dissipates and we observe an essentially straight jet, excepting some initial curvature in the supersonic region close to triple points.

In all cases the initial jet curvature is expected to be upstream, towards the main advancing detonation front. This includes the small jet studied in the inset of figure 9 that appears contrary to this prediction. Close examination reveals that the initial curvature right at the point of jet formation, is indeed towards the main detonation front, before it is overwhelmed by the influence of the global flow field. Also obscuring the initial curvature is an angular misalignment of the initial wave angles, due to sensitivity of the solution to local variations in track angle and overdrive as discussed in section 4 .

\section{Conclusions}

We have shown that under transformation to a common frame of reference fixed with respect to the primary shock intersection point and aligned with the incident flow direction, the phenomena of unstable detonation propagation and steady hypervelocity flow blunt body shock wave interaction share a common shock structure. In both cases local satisfaction of the conservation laws at bifurcation points, on the otherwise smooth shock fronts, demands the local existence of a supersonic jet structure embedded in the surrounding transonic flow, irrespective of the distortion of the bulk flow necessary to accommodate its existence.

Local analysis about the shock wave intersection points with simple thermochemical models shows that all wave strengths and thermodynamic properties in the vicinity of the near-field jet structure are completely determined by the mixture, initial conditions, and by the assumption of both global and local satisfaction of the Chapman-Jouguet hypothesis by the overall system and the local detonation stem of the transverse wave system respectively (in the case of self sustaining waves as observed in strong mixtures). Non-self sustaining solutions (observed in weak mixtures) require external knowledge of the far field flow to close the near field jet solution. Reflecting the importance of any diluent in determining the gas-dynamic characteristics of the detonation, we show that the jetting phenomenon is both weaker and 
non-self propagating for mono-atomic diluents in comparison with diatomic diluents.

The multiple oblique shock waves of the jet produce less entropy than the strong main detonation front and the transverse detonation stem. Since temperature reduces with entropy at the nearly constant pressure of the downstream transonic layer, the chemical kinetic rates are suppressed for fluid that passes through the jet shock system. Thus we have demonstrated a mechanism for the formation of the bulk unreacted gas pockets observed in strongly unstable detonation, with potentially distinct reaction pathways, in the region downstream of the advancing portion of the main detonation front. We show that the jetting mechanism is consistent and highly synergistic with prior kinetic and stability studies for complex mixtures whereby the reduced effective activation energy and the ratio of induction to energy release time scales arise as key parameters.

Comparison with existing data for representative mixtures shows good agreement between overlaid predictions of wave angles and Schlieren images and also with the locations and form of unreacted gas pockets in both $\mathrm{OH}$ PLIF images and CFD species predictions for complex gas mixtures. Predictions of local detonation track angle vs. local triple point propagation velocity have been validated against CFD predictions (that do not simulate the wall effects of small tubes and thin channels). Reflecting the highly constrained nature of the local solution, we document the persistence of the fundamental jet shock structure over a wide range of length scales in the data. In many cases the jet structure is sufficiently compressed that it superficially appears as a single point shock interaction. In the far field, where the current local model does not apply, we highlight different wave structures produced in the cases of weak and strong instability along with the influence of the relative transverse propagation speeds of the far-field transverse wave, the adjoining transverse detonation stem, and the near field jet structure.

\section{Acknowledgment}

The five junior authors wish to express their gratitude to Prof. Hornung for his enthusiastic guidance and friendship over a period of many years. The citations in this paper clearly articulate the powerful influence that Hans' prior studies have exerted on the direction of the current investigation. This work could never have begun without his leadership of the GALCIT community and his support for our privileged membership of it. We happily 
dedicate this manuscript to the celebration of his 75 th birthday.

\section{References}

[1] Austin JM. The role of instability in gaseous detonation. Caltech Ph.D. Thesis, 2003.

[2] Austin JM, Pintgen F, Shepherd JE. Reaction zones in highly unstable detonations. Combustion Institute 2005; 30:1849-1857.

[3] Shepherd JE. Detonations in gases. Combustion Institute 2009; 32:8398.

[4] Radulescu MI, Sharpe GJ, Law CK, Lee JHS. The hydrodynamic structure of unstable cellular detonations. Journal of Fluid Mechanics 2007; 580:31-81.

[5] Pintgen F, Eckett CA, Austin JM, Shepherd, JE. Direct observations of reaction zone structure in propagating detonations. Combustion and Flame 2003; 133(3):211-229.

[6] Eckett CA, Quirk JJ, Shepherd JE. The role of unsteadiness in direct initiation of gaseous detonation. Journal of Fluid Mechanics 2000; 421:147183.

[7] Massa L, Austin JM, Jackson TL. Triple point shear layers in gaseous detonation waves. Journal of Fluid Mechanics 2007; 586:205-248.

[8] Sanderson SR. Shock wave interaction in hypervelocity flow. Caltech Ph.D. Thesis, 1995.

[9] Sanderson SR, Hornung HG, Sturtevant B. The influence of nonequilibrium dissociation on the flow produced by shock impingement on a blunt body. Journal of Fluid Mechanics 2004; 516:1-37.

[10] Edney BE. Anomalous heat transfer and pressure distributions on blunt bodies at hypersonic speeds in the presence of an impinging shock. FFA Report 115, 1968.

[11] Edney BE. Effects of shock impingement on the heat transfer around blunt bodies. AIAA Journal 1968; 6(1):15-21. 
[12] Sanderson SR. Gasdynamic wave interaction in two spatial dimensions. Journal of Fluid Mechanics 2004; 506:187-205.

[13] Sanderson SR, Sturtevant B. Shock wave interactions in hypervelocity flow. Proc. 19th International Symposium on Shock Waves, Marseille, 1993.

[14] Brück S. Investigation of shock-shock interactions in hypersonic reentry flows. Proc. 20th Int. Sym. Shock Waves, Pasadena, 1995.

[15] Carlson AB, Wilmoth RG. Monte Carlo simulation of a near continuum shock-shock interaction problem. Journal of Spacecraft and Rockets, 1994; 31(1):25-30.

[16] Hornung HG. Regular and Mach reflection of shock waves. Annual Review of Fluid Mechanics 1986; 18(33):33-58.

[17] Liang Z, Browne S, Deiterding R, Shepherd JE. Detonation front structure and the competition for radicals. Combustion Institute 2007; $31: 2445-2453$.

[18] Voitsekhovskii BV, Mitrofanov VV, Topchian ME. Struktura fronta detonastii i gaza. Akad. Nauk., SSSR, Novosibirsk, 1963, Translation: The structure of a detonation front in gases. Rep. FTD-MT-64-527, Foreign Technology Division, Wright-Patterson A.F.B., Ohio, 1966.

[19] Subbotin V. Two kinds of transverse wave structures in multi-front detonation. Fizika Goreniya i Vzryva 1975; 11(1):96-102. Translation: Combustion, Explosion, and Shock Waves 1975; 11(1):83-88.

[20] Oppenheim A, Smolen J, Zajac L. Vector polar method for the analysis of wave interactions. Combust. Flame 1968; 12(1):63-76.

[21] Urtiew P. Reflections of wave intersections in marginal detonations. Astronautica Acta 1970; 15:335-343.

[22] Barthel HO. Reaction zone-shock front coupling in detonations. Physics of Fluids 1972; 15(1):43-50.

[23] Sanderson SR, Hornung HG, Sturtevant B. Aspects of planar, oblique and interacting shock waves in an ideal dissociating gas. Physics of Fluids 2003; 15(6):1638-1649. 
[24] Courant R, Friedrichs KO. Supersonic flow and shock waves. Interscience, New York, 1948.

[25] Hornung HG, Oertel H, Sandeman RJ. Transition to mach reflexion of shock waves in steady and pseudosteady flow with and without relaxation. Journal of Fluid Mechanics 1979, 90(3):541-560.

[26] Lighthill MJ. Dynamics of a dissociating gas, part I, equilibrium flow. Journal of Fluid Mechanics 1957; 2(1):1-32.

[27] Freeman NC. Non-equilibrium flow of an ideal dissociating gas. Journal of Fluid Mechanics 1958; 4(4):407-425.

[28] Mouton CA, Hornung HG. Mach stem height and growth rate predictions. AIAA Journal 2007; 45(8):1977-1987.

[29] Mouton CA, Hornung HG. Experiments on the mechanism of inducing transition between regular and Mach reflection. Physics of Fluids 2008; 20:126103.

[30] Reynolds WC. The element potential for chemical equilibrium analysis: implementation in the interactive program STANJAN. Technical Report A-3991, Department of Mechanical Engineering, Stanford, CA, 1986.

[31] Konnov A. Detailed reaction mechanism for small hydrocarbon combustion. http://homepages.vub.ac.be/akonnov, Release 4.0.

[32] Warnatz J, Karbach V. C2 mechanism for methane-air combustion. http:www.ca.sandia.gov/tdf/3rdWorkshop/ch4mech.html. 